\title{
Magnetic and Electronic Properties of Heavy Lanthanides (Gd, Tb, Dy, Er, Ho, Tm)
}

\author{
Radel R. Gimaev ${ }^{1}$, Aleksei S. Komlev ${ }^{1}\left(\mathbb{D}\right.$, Andrei S. Davydov ${ }^{2}{ }^{(}$, Boris B. Kovalev ${ }^{1,3}$ and Vladimir I. Zverev ${ }^{1, *(\mathbb{D})}$ \\ 1 Faculty of Physics, M.V. Lomonosov Moscow State University, 119991 Moscow, Russia; \\ gimaev@physics.msu.ru (R.R.G.); komlev.as16@physics.msu.ru (A.S.K.); bbkovalev@gmail.com (B.B.K.) \\ 2 Burnazyan Federal Medical Biophysical Center, 123182 Moscow, Russia; davydov.andrey@physics.msu.ru \\ 3 National Research Center "Kurchatov Institute", 123182 Moscow, Russia \\ * Correspondence: vi.zverev@physics.msu.ru
}

check for updates

Citation: Gimaev, R.R.; Komlev, A.S. Davydov, A.S.; Kovalev, B.B.; Zverev, V.I. Magnetic and Electronic Properties of Heavy Lanthanides (Gd, Tb, Dy, Er, Ho, Tm). Crystals 2021, 11, 82. https://doi.org/10.3390/ cryst11020082

Academic Editor:

Alessandro Chiasera

Received: 5 January 2021

Accepted: 20 January 2021

Published: 21 January 2021

Publisher's Note: MDPI stays neutral with regard to jurisdictional claims in published maps and institutional affiliations.

Copyright: (c) 2021 by the authors. Licensee MDPI, Basel, Switzerland. This article is an open access article distributed under the terms and conditions of the Creative Commons Attribution (CC BY) license (https:// creativecommons.org/licenses/by/ $4.0 /)$.

\begin{abstract}
Rare earth metals (REM) occupy a special and important place in our lives. This became especially noticeable during the rapid development of industry in the industrial era of the twentieth century. The tendency of development of the rare-earth metals market certainly remains in the XXI century. According to experts estimates the industry demand for chemical compounds based on them will tend to grow during the nearest years until it reaches the market balance. At the same time, the practical use of high-purity rare-earth metals requires the most accurate understanding of the physical properties of metals, especially magnetic ones. Despite a certain decline in interest in the study of high-purity REM single crystals during the last decade, a number of scientific groups (Ames Lab, Lomonosov Moscow State University (MSU), Baikov Institute of Metallurgy and Materials Science Russian Academy of Science (RAS)) are still conducting high-purity studies on high-purity metal samples. The present article is a combination of a review work covering the analysis of the main works devoted to the study of heavy REMs from gadolinium to thulium, as well as original results obtained at MSU. The paper considers the electronic properties of metals in terms of calculating the density of states, analyzes the regularities of the magnetic phase diagrams of metals, gives the original dependences of the Neel temperature and tricritical temperatures for Gd, Tb, Dy, Er, Ho, Tm, and also introduces a phenomenological parameter that would serve as an indicator of the phase transformation in heavy REMs.
\end{abstract}

Keywords: rare earth; magnetism; DOS; Gd; Tb; Dy; Ho; Er; Tm; magnetic phase diagram; phase transitions

\section{Introduction}

The family of lanthanides (rare earth metals) includes 13 or 15 elements, from cerium to ytterbium or from lanthanum to lutetium. In the Periodic Table of elements, they are placed between barium and hafnium, so REMs usually have a separate row at the bottom of the table. From the very meaning of Mendeleev's table, one would think that all REMs have exactly the same properties. However, this was a mistake because the technology for obtaining these substances in pure form was virtually non-existent. REMs were produced only in the form of complex similar compounds, which created the illusion of their physical and chemical similarity. When REM became available in a sufficiently pure form, it turned out that the differences between them can be as great as those between other chemical elements. For this reason, intensive research on REM began only in the 1950s. Today, these metals are widely used in almost all industries and, most importantly from a scientific point of view, serve as model objects for testing and refining various physical theories.

At present, it cannot be claimed that a complete theory of pure metallic REM atoms or ions, much less their crystals or alloys and compounds, has been created, although many theoretical approaches to explain various characteristics of REM crystals have been proposed. First theoretical works on heavy REMs were published in the 1960s [1-3]. 
They were continued for the spin-wave spectrum in [4]. A theoretical study of the effects of anisotropy on spiral spin-configurations, emphasizing the problem of the magnetic ordering in rare-earth metals has been conducted in [5]. Fermi surfaces and spin structures have been considered in [6]. One of the theoretical attempts to sum the experimental material up has been done in [7] where using Gd, as a prototype for all heavy rare-earth metals, the authors have generated a unified magnetic phase diagram, which links the magnetic structures of the metals to their lattice parameters. Fermi-surface topology and helical antiferromagnetic ordering in lanthanide metals have been studied in [8]. The standard model of the rare earths from Hubbard approximation I are analyzed in [9]. The latest up-to-date theoretical ab initio studies of the magnetic field and temperature-induced transitions between long-period helical antiferromagnetic (AFM), fan, and ferromagnetic (FM) phases in the heavy lanthanide elements [10-12]. At the same time, there is still no theory that reliably predicts the presence of magnetic ordering in REM, especially noncollinear ordering. All existing approaches are, as a rule, phenomenological or ad hoc in nature.

At the same time, a huge and diverse experimental material on the study of the physical and chemical properties of these substances has been collected. The amount of published works is so big [13-22] (for example, one can find about 100 works devoted to each of the heavy REMs, except for thulium), which makes it impossible to give a detailed review of the content of each of them.

For the first time here, the magnetic phase diagrams of metals are studied for the whole series of heavy REM, and for gadolinium, terbium, holmium, and dysprosium they were obtained by one of the authors, while the data for erbium and thulium are taken from the latest literature sources. The peculiarity of consideration is the choice of single-crystalline samples of heavy REM, which is quite rare nowadays due to the high cost of production of high-purity single crystals and certain technological difficulties in their production. At the same time, the consideration of all diagrams together in this work was done for the first time, which allowed to reveal the general regularities of magnetic ordering in heavy REM. Moreover, the calculation of the density of states of heavy REM, performed for the first time using the projector-augmented waves (PAW) method, combining the ideas of the pseudopotential method and LAPW, is original. This method is also applicable to the influence of impurities on the electronic properties of REM, which will be shown by the authors in a separate paper. In addition, the original results are also the establishment of a mutually unambiguous relationship between the values of the Neel temperature of heavy REMs and de Gennes function, as well as the general behavior of the magnetic heat capacity maximum temperature in all heavy REMs.

\section{Theoretical Consideration}

The nature of magnetic ordering in heavy REM is explained by an indirect exchange mechanism (Ruderman-Kittel-Kasuya-Yosida (RKKY) interaction) [23,24]. Spins of conduction electrons are polarized by magnetic atoms by means of localized exchange interaction, which results in a cloud of spin density concentrated near the magnetic atom. As the distance from the magnetic atom increases, the spin density decreases according to one law or another. The second magnetic atom interacts with the first through this induced spin density. The s-d exchange model played an essential role in the theory of indirect exchange [25]. In particular, it has been shown that the total Hamiltonian of the system contains a term describing the exchange interaction between the localized d-electron and the delocalized s-electron. Since the additional s-d exchange term in the Hamiltonian is small compared with the other terms, one can apply the method of perturbation theory. Already in the second-order of the theory, it appears that there is an effective exchange relation between magnetic ions through conduction electrons: 


$$
\mathcal{J}_{\text {eff }}(\vec{R})=-\frac{1}{V^{2}} \sum_{k, k^{\prime}}^{\prime} \frac{\left|\mathcal{J}\left(\vec{k}, \overrightarrow{k^{\prime}}\right)\right|^{2}}{E_{k}-E_{k^{\prime}}} \exp \left[i\left(\overrightarrow{k^{\prime}}-\vec{k}\right) \vec{R}\right] f\left(E_{k}\right)
$$

where $\mathcal{J}\left(\vec{k}, \overrightarrow{k^{\prime}}\right)$ is the exchange integral, $\vec{k}$ is the wave vector of s-electron, $\vec{R}$-radius-vector of the ion, $f\left(E_{k}\right)$-the Fermi distribution function. The dash at the sum means that the summation omits the terms with $\vec{k}=\overrightarrow{k^{\prime}}$.

An explicit form of the exchange integral can be obtained by assuming the constancy of the elementary integral $\mathcal{J}\left(\vec{k}, \overrightarrow{k^{\prime}}\right)$. By replacing the variables $\overrightarrow{k^{\prime}}-\vec{k}=\vec{q}$, one can obtain:

$$
\mathcal{J}_{\text {eff }}(\vec{R})=\frac{\mathcal{J}^{2}}{V^{2}} \sum_{q \neq 0} \sum_{k} \frac{f\left(E_{k}\right)}{E_{k+q}-E_{k}} \exp [i \vec{q} \vec{R}]
$$

If one assumes that the conduction band is almost empty (almost free electrons approximation), then $E_{k}=\frac{\hbar^{2} k^{2}}{2 m}$ и

$$
E_{k+q}-E_{k}=\frac{\hbar^{2}}{2 m} \vec{q}(2 \vec{k} \cos \Theta+\vec{q})
$$

where $\Theta$ is the angle between the vectors $\vec{k}$ и $\vec{q}$.

From Equations (2) and (3) one can get

$$
\mathcal{J}_{\text {eff }}(\vec{R})=\frac{\mathcal{J}^{2}}{V^{2}} \frac{2 m}{\hbar^{2}} \sum_{q \neq 0} \frac{\exp [i \vec{q} \vec{R}]}{\vec{q}} \sum_{k} \frac{f\left(E_{k}\right)}{2 \vec{k} \cos \Theta+\vec{q}}
$$

Using the spherical coordinate system (except for the angle $\Theta$ one can introduce $\alpha$ which is the angle between $\vec{R}$ and $\vec{q}$ ) and replacing the summation by integration, in the low-temperature approximation, when the Fermi distribution can be considered rectangular, one can obtain

$$
\mathcal{J}_{\text {eff }}(\vec{R})=\frac{m \mathcal{J}^{2}}{8 \pi^{3} \hbar^{2} R^{2}} \frac{1}{4 R^{2}}\left(\sin 2 k_{F} R-2 k_{F} R \cos 2 k_{F} R\right)
$$

where $k_{F}$ is the value of the wave vector on the Fermi surface. If one introduces the Ruderman-Kittel function in the form:

$$
F(x)=(x \cos x-\sin x) / x^{4}
$$

then

$$
\mathcal{J}_{\text {eff }}(\vec{R})=-\frac{\mathcal{J}^{2}}{E_{F}} \frac{k_{F}^{6}}{4 \pi} \frac{V^{2}}{N^{2}} F\left(2 k_{F} R\right)
$$

Formulae (5) and (7) are two variants for RKKY interaction. It is characterized by two features: it is long-range and its amplitude decreases according to the power-law; the interaction is oscillatory, sign-variable, i.e., a particular magnetic ion can be connected with its distant neighbors both ferro- and antiferromagnetically. Determining the specific type of interaction in a given rare-earth metal requires taking into account the interaction of the ion with several neighbors of varying degrees of remoteness. The total electron energy due to the exchange interaction looks more complex than the equation for the RKKY interaction obtained in the second order of perturbation theory [26].

The exchange interaction between conduction electrons and $4 \mathrm{f}$-electrons depends on the orientation of the resultant spin of the unoccupied 4f-layer of the REM ion. The helicoidal and sinusoidal arrangement of the magnetic moments of the ions is characterized by a periodic change in the spin projection on the hexagonal c-axis or an axis perpendicular 
to it. As a consequence, the energy of the conduction electrons is periodic with a period equal to the period of the magnetic superlattice, which is much greater than the period of the crystal lattice. As magnetic order arises and increases, the Fermi surface will continuously deform. The effects of strong changes in the Fermi surface due to the formation of helicoidal or sinusoidal structures at temperatures below the Neel temperature have been considered in detail and quantified by Dzyaloshinskii $[27,28]$. These effects should be considered when explaining the very appearance of spiral ordering. The specifics of the Fermi surface in heavy REMs determine the character of the magnetic structure immediately below the magnetic ordering temperature. In the course of calculations of the Fermi surface of heavy REMs [29,30] the presence of the so-called "bridges" of the full Fermi surface was discovered. In [31] it was shown that the change in the thickness of this "bridge" from one metal to another provides the correct trend in the behavior of the magnetic properties of REM; in particular, the absence of such a bridge can explain why gadolinium does not have a periodic ordering. The numerical results obtained so far, as well as the absence of a rigorous theory of rare-earth magnetism, allow only to say that the properties of the Fermi surface play a fundamental role in establishing periodic magnetic structures in heavy REMs.

The magnetic structure is significantly transformed when the temperature decreases due to changes in the energy spectrum of conduction electrons and due to the influence of magnetocrystalline and magnetoelastic interactions [26]. Polarization of conduction electrons also strongly changes in REM as a result of magnetic transitions. Thus, magnetic ordering and electronic structure in heavy REM are interrelated.

In this paper, based on the method proposed in [32], densities of states for all heavy REM were calculated (Figure 1). The Quantum ESPRESSO utility, an integrated set of opensource computer programs for calculating electronic structures and modeling nanoscale materials based on density functional theory (DFT), plane waves (PW), and pseudopotentials, was used to calculate the density of states. A unit cell of two atoms was used to calculate the electronic structures of chemical elements with an hcp crystal lattice. The parameters of the crystal lattice a and c were taken from [33]. Calculations were performed using the projector-augmented waves (PAW) method [34,35], combining the ideas of the pseudopotential method and Linearized Augmented Planewave (LAPW) [36]. The method reproduces the exact wave function of the valence electrons, combining the flexibility of the pseudopotential method with the accuracy of the full potential methods. In the calculations of the exchange-correlation (XC) energy functional the local density approximation (LDA) was used, which depends exclusively on the electron density value at each point in space (and not, for example, on density derivatives or Kohn-Sham orbitals [37]). The specific implementation of LDA is taken from [38]. To calculate the density of states (DOS), it is necessary to calculate the Brillouin zone integrals. For this purpose, it is necessary to replace them with a weighted sum over special points in k-space. The number of points in k-space (i.e., k-grid) in which the Brillouin zone is discretized determines the accuracy of the calculation. The choice of the k-point grid depends on the problem under consideration. More grid points (smaller grid interval) gives better total energy convergence. Here the Brillouin zone is discretized using the scheme proposed in [39], to obtain a grid with equal intervals. The DOS calculation was performed in two stages. First, a self-consistent charge density was calculated with a small number of points in the k-lattice, then nonself-consistent calculations were performed using an already fixed self-consistent charge density. The use of an $8 \times 8 \times 6$ face-centered k-lattice proved to be sufficient to achieve acceptable accuracy. The kinetic energy cutoff parameters for the wave functions were chosen equal to $60 R y$, for charge density and potential-240 Ry. 

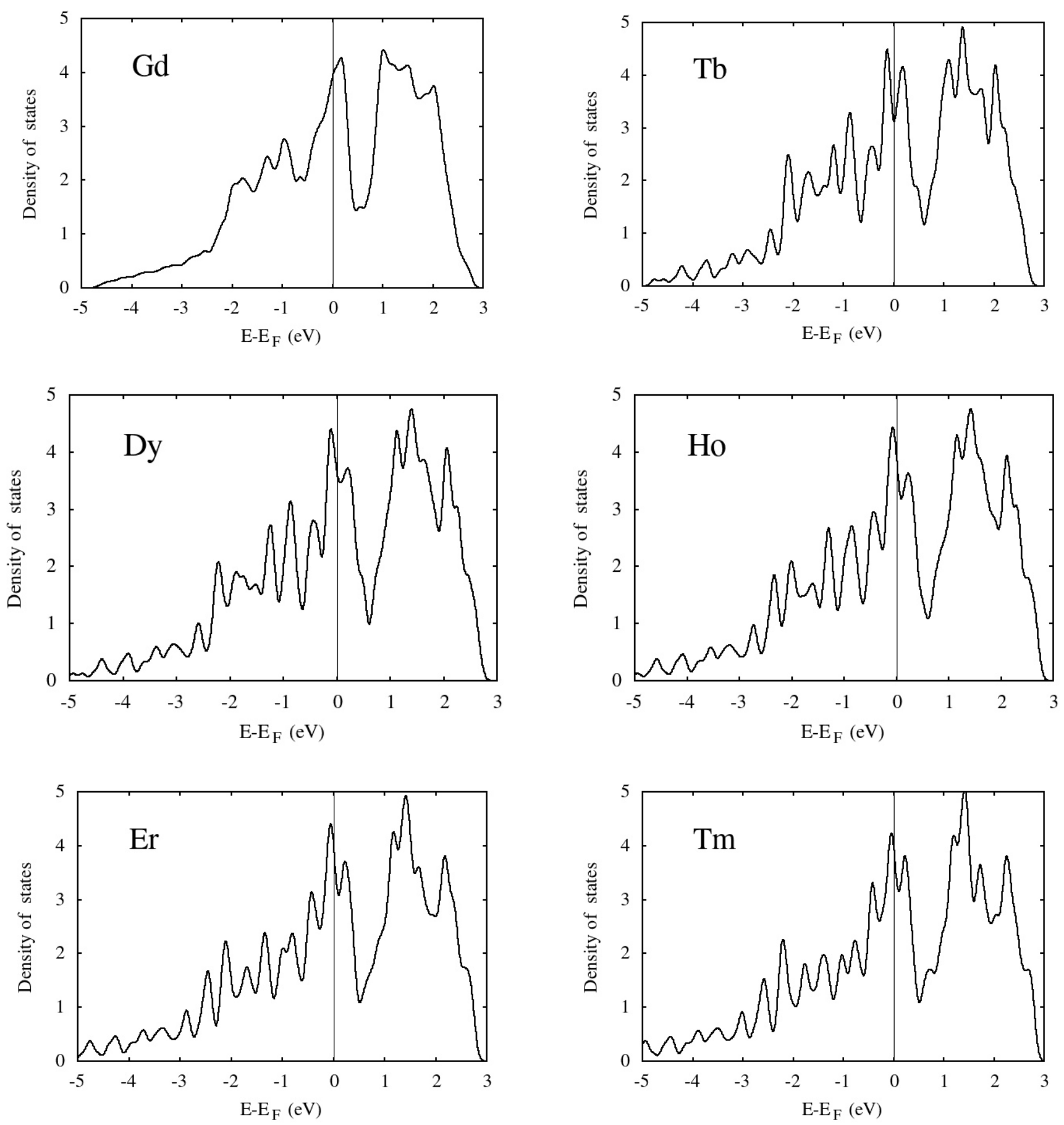

Figure 1. The density of states (DOS) of heavy lanthanides Gd, Tb, Dy, Ho, Er, Tm.

The plots show that at $\mathrm{E}=\mathrm{E}_{\mathrm{F}}$ the DOS value is maximal or close to maximal. This insignificant change in the Fermi energy is because the concentration of free electrons per unit volume changes weakly in the series of heavy REM from gadolinium to thulium. In [40] it was predicted that the periodic electronic structure caused by the s-f interaction, which determines the magnetic properties of the considered metals, affects the shape of the Fermi surface. Self-intersections of the Fermi surface take place even in the case of infinitesimal perturbation. Thus, the exchange interaction, by removing degeneracy, leads to the splitting of the peaks in the figure $n$ and to the formation of non-intersecting cavities of the Fermi surface. It also follows from the aforementioned work that the half-width of the energy gap between peaks is proportional to the integral of the s-f interaction and to 
the mean value of the magnetic moment of the f-electrons. This fact explains the strong difference of the DOS curve of gadolinium from the other elements. The total orbital momentum of gadolinium is 0 , and the spin has the highest value compared to the other elements $(7 / 2)$. Therefore, the weak spin-orbital interaction in gadolinium leads to the observed absence of split peaks in Figure 1 and to their significant broadening due to the maximum value of the average magnetic moment per electron. The calculated density of states for gadolinium is in good agreement with previously published data [41-43]. Gd has strongly correlated electrons, with a split between spin-up and spin-down f-bands. Gd is at half-filling of f-band. Without proper consideration of strongly correlated f-electrons, even magnetic moment per Gd atom is incorrect in DFT. Subtle differences in the wave functions of conduction electrons and their energy bands in different metals lead to a variety of magnetic structures and properties of these metals.

Theoretical investigation of the problem of magnetic ordering in REM is also carried out within the framework of the molecular field model [3,5,44-46]. It correctly describes important properties of ferromagnets, such as the presence of spontaneous magnetization and its temperature dependence, the high-temperature behavior of heat capacity, and magnetic susceptibility [47]. When the nature of the initial Hamiltonian and the interaction are properly accounted for, it is possible to use this theory to obtain magnetic structures that correspond to the experimental data, as well as to determine the boundaries between the phases. At the same time, it does not accurately describe the magnetization at low temperatures and in the vicinity of the Curie temperature. In addition, this theory predicts a reversal to zero of the magnetic part of the heat capacity above the Curie temperature, which does not correspond to reality. At the same time, this model is applicable to describe the magnetic properties of REM because of the strong atomic localization of f-electrons.

The complete spin Hamiltonian can be represented as

$$
\hat{\mathcal{H}}=\hat{\mathcal{H}}_{e x}+\hat{\mathcal{H}}_{c f}+\hat{\mathcal{H}}_{m e}
$$

where the different terms describe the following contributions: $\hat{\mathcal{H}}_{e x}$-exchange energy, $\hat{\mathcal{H}}_{c f}$-crystal field, $\hat{\mathcal{H}}_{m e}$-magnetoelastic energy.

The exchange contribution can be represented as

$$
\hat{\mathcal{H}}_{e x}=-\sum_{i \neq j} \dot{\mathcal{L}}\left(\vec{R}_{i}-\vec{R}_{j}\right) \hat{s}_{i} \hat{s}_{j}
$$

where $j\left(\overrightarrow{R_{i}}-\overrightarrow{R_{j}}\right)$ is exchange interaction between spins localized at nodes $\vec{R}_{i}$ и $\vec{R}_{j}$, and $\hat{s}_{i}$, $\hat{s}_{j}$ are the corresponding total spin operators. Overlaying this interaction on the set of states of ions renumbered using the quantum number of the total momentum, one can obtain:

$$
\hat{\mathcal{H}}_{e x}=-\left(g_{J}-1\right)^{2} \sum_{i \neq j} \dot{\mathcal{L}}\left(\vec{R}_{i}-\vec{R}_{j}\right) \hat{J}_{i} \hat{J}_{j}=-\sum_{i \neq j} \mathcal{J}_{i j} \hat{J}_{i} \hat{j}_{j}
$$

where $\hat{J}_{i}=\hat{L}_{i}+\hat{S}_{i}$ is is the total-momentum operator of the $\mathrm{i}$-th ion. The microscopic theory gives in the simplest form:

$$
\mathcal{J}\left(\vec{R}_{i}-\vec{R}_{j}\right)=\frac{2}{N^{2}} \sum_{q}|U(\vec{q})|^{2} \chi(\vec{q}) \exp \left[i\left(\vec{R}_{i}-\vec{R}_{j}\right) \vec{q}\right]
$$

where $\chi(\vec{q})$ is the generalized susceptibility of conduction electrons (response function), $\mathrm{N}$ is the total number of unit cells in the crystal, $U(\vec{q})$ is the matrix element of the average energy between the $\mathrm{f}$ - and s-electrons.

Equation (10) describes isotropic exchange. Anisotropy can result from several mechanisms [48]. Firstly, because of the dependence of the s-f matrix elements on the orbital states of the f-electrons. Estimates show that this effect does not exceed $10 \%$ of the average 
exchange energy. Secondly, in ordered phases, the polarization of conduction electrons results in splitting between the longitudinal and transverse components of $\mathcal{J}_{i j}$. This effect is small because the exchange splitting of the conduction zone is $\sim 10^{-4}$ of the typical zone width. A similar contribution is made by the spin-orbital interaction of the conduction electrons. Another, very small, contribution may be made by the modification of the s-f exchange matrix element by the spin-orbital interaction of conduction electrons [49] or indirect exchange through spin-orbital interaction of conduction electrons [50]. Although the individual contributions of the different anisotropy mechanisms are small, their sum may be significant enough to result in appreciable anisotropic effects.

The terms describing the interaction with the crystal field can be included by decomposing the electrostatic potential around the lattice node occupied by the REM ion into a series of spherical harmonics and passing from spherical harmonics to equivalent orbital moment operators by the Wigner-Eckart theorem. The number of members of the series is limited by the point group of symmetry of the node and the orbital momentum of the f-electron $(l=3)$. The general equation for the Hamiltonian describing the crystal field contribution can be written as

$$
\hat{\mathcal{H}}_{c f}=\sum_{i} \sum_{l m} B_{l m} \theta_{l}^{m}\left(J_{i}\right)
$$

Here the first summation is performed on nodes $i$, and $\theta_{l}^{m}\left(J_{i}\right)$ are operators equivalent to the corresponding spherical harmonics obtained by replacing the Cartesian components of spherical harmonics with Cartesian components of $J$,

$$
B_{l m}=\alpha_{l}\left\langle r^{l}\right\rangle A_{l m}
$$

where $\alpha_{l}$ is the Stevens factor, which depends on the specific environment of the ion, $\left\langle r^{l}\right\rangle$ means the probable value according to the radial distribution, $A_{l m}$ are the crystal field parameters, which can be calculated from the charge distribution around the ion.

In many cases magnetic ordering can be accompanied by magnetostrictive stresses, which modify the crystal field, lowering the ion energy. The resulting magnetoelastic contributions are usually written in terms of equilibrium stresses and magnetostrictive coefficients [51].

Various magnetic structures observed in REM arise as a result of the combined action of the three types of energy discussed above: exchange energy, crystal field, and magnetoelastic energy. The exchange energy plays a decisive role in the case of noncollinear structures, since the Ruderman-Kittel function is sign-variable, and the force which it determines is long-range. Other energies tend to be localized in the lattice, determining moment orientation and leading to ferromagnetism, or at least commensurability of the crystal and magnetic structures. Magnetic ordering in heavy REMs can be derived from the molecular field model [44,46,51].

In general terms, all structures can be described by the following equation:

$$
\overrightarrow{\mathcal{J}}_{l}=\sum_{\alpha} \overrightarrow{\mathcal{J}}_{q \alpha} \exp \left[i \overrightarrow{q_{\alpha}} \vec{R}_{l}\right]
$$

where the summation is carried out for all periods $\alpha$ (with the corresponding wave vectors $\overrightarrow{q_{\alpha}}$ ), which exist simultaneously, and the vector amplitudes $\overrightarrow{\mathcal{J}_{q \alpha}}$ are complex. The general relation (14) includes ferromagnetism with moments lying in the basis plane; conical spirals modulated along the c-axis (including the limiting case of pure modulation described by a longitudinal spin density wave); ferromagnetic structures directed along the c-axis. 
In phenomenological theory, it is more convenient to write these structures as functions of the angle $\Theta$ between neighboring moments along the axis:

$$
\begin{gathered}
\overrightarrow{\mathcal{J}}_{l \xi}=\vec{s} \cos \theta \\
\overrightarrow{\mathcal{J}}_{l \zeta}=\vec{s} \sin \theta \cos \left(\vec{q} \overrightarrow{R_{l}}\right) \\
\overrightarrow{\mathcal{J}_{l \eta}}=\vec{s} \sin \theta \sin \left(\vec{q} \overrightarrow{R_{l}}\right)
\end{gathered}
$$

Axes $\xi, \eta$, and $\zeta$ are chosen in the hexagonal system so that the axis $\zeta$ is directed along the axis $c$ of the crystal, the axis $\xi$ is the easy magnetization axis in the basal plane, $\eta$ is perpendicular to $\xi$. In this case, relations (15) do not describe the ferrimagnetic structure and spin waves.

In molecular field theory, the exchange energy per ion is obtained from Equation (10) as:

$$
E_{e x} / N=-\mathcal{J}(q) \vec{s}^{2} \sin ^{2} \theta-\mathcal{J}(0) \vec{s}^{2} \cos ^{2} \theta
$$

where $\mathcal{J}(\vec{q})=\sum \mathcal{J}_{i j} \exp \left[i \vec{q}\left(. \vec{R}_{i}-\vec{R}_{j}\right)\right]$.

From Equation (16) one can see that the system will choose such a value of $\vec{q}_{m}$ as the magnetic period, for which $\mathcal{J}(\vec{q})$ is minimal.

Minimizing energy at a given value $\vec{q}_{m}$ considering also the axial anisotropy leads to the following values of the angle $\Theta$ :

$$
\begin{gathered}
\sin \theta=0-\text { uniaxial ferromagnetic } \\
\cos \theta=0-\text { spiral in the basal plane } \\
3 V_{6} \vec{s}^{4} \cos ^{4} \theta+2 V_{4} \vec{s}^{2} \cos ^{2} \theta+\left[\mathcal{J}\left(\vec{q}_{m}\right) \cdot-\mathcal{J}(0)+V_{2}\right]=0
\end{gathered}
$$

The latter equation describes the cone structure in general; the coefficients describe the axial anisotropy of the crystal and are calculated from the equations for the crystal field. Stability conditions for these structures are discussed in [4]. At high temperatures, the member containing $V_{2}$, is maximal, the magnitude and sign of $V_{2}$ determine whether the moments lie in the basal plane $\left(V_{2}>0\right)$, whether they form a cone structure or are located along the hexagonal axis, which corresponds to a ferrimagnetic structure or a longitudinal wave of spin density $\left(V_{2} \ll 0\right)$.

Regular spiral or cone structures are obtained if one neglects anisotropy in the basal plane and magnetoelastic effects when calculating energy. Accounting for these relatively small influences can lead to helicity modification, the formation of "bunches" of spins near-certain directions. This phenomenon was discovered in neutron diffraction, and later a theory was proposed for the case of a flat helix [52]. A similar consideration, taking into account the magnetoelastic effects, can be carried out for the general case of a conical structure [53]. When a magnetic field is applied to spiral or conical structures, distortion with a small excess moment along the field occurs. In fields exceeding the first critical value (at a given temperature), a fan phase emerges, in which moments are located near the field direction, and in fields exceeding the second critical value, the fan phase breaks down, passing into collinear ferromagnetic [2]. If the anisotropy in the basis plane is large enough, the fan phase may not occur.

In general, the magnetic and crystal structures of rare-earths are incommensurable with each other. However, because the periods of these structures change unequally with temperature, there are situations when the commensurability can take place. The reasons leading to the transition to the commensurable phase are as follows:

- axial anisotropy, together with the magnetoelastic forces, favors the maximum momentum at each node for a given configuration of exchange forces, which can only be achieved in a commensurate structure;

- exchange interaction also favors the maximum momentum, providing a change with a periodicity not inconsistent with the maximum $\mathcal{I}(q)$. At higher temperatures, 
increasing entropy in the incommensurable phase likely plays a role in maintaining incommensurability $\vec{q}_{m}$ and the development of instability at potential transition points, preventing the occurrence of a phase transition.

With a further decrease in temperature, the growth of the anisotropic terms of the Hamiltonian leads to phase transitions of the first order to other structures as in terbium and dysprosium a planar spiral structure undergoes the transition to a ferromagnetic phase with the of easy magnetization direction lying in the basal plane. Magnetostrictive stresses play a decisive role in this transition.

In some cases, a conical structure (ferromagnetic spiral) arises at low temperatures. The conditions for the emergence of such a phase were considered within the framework of the molecular field model [4]. It was shown that the reason for the transition to a conical structure lies in the peculiarities of the temperature dependence of the axial anisotropy coefficients. In the case of a sufficiently slow temperature change of the anisotropy coefficients near the helix-cone transition temperature, the instability can lead to the appearance of spin waves with a wave vector close to $\vec{q}_{m}$, which, in turn, leads to an intermediate phase between the spiral and the cone [53]. As it is shown in [54], this intermediate phase is a canted spiral [55].

In pure REMs, other intermediate phases are observed that are quite simply described, for example, by a superposition of spiral and longitudinal spin density wave structures with equal periods. Such a phase occurs, in particular, in erbium. The calculation of the magnetic structure of erbium was performed within the framework of the molecular field model [56], both the effective crystal field parameters and the interplanar two-ion interaction parameter were taken into account. The parameters were estimated from the experimentally obtained values of transition points and magnetization. The details of the obtained magnetic structure were compared with the results of neutronography [57]. The calculation predicted the real existing structures, although there were quantitative discrepancies with the experiments.

The molecular field method cannot be applied to strictly describe the properties of magnetics in the two limiting regions-near the points of phase transitions and at low temperatures. At the same time, the low-temperature properties of heavy REMs are could be determined by spin waves' theory [58]. When applied to rare-earth metals, it (both for the case of ferro- and antiferromagnetic ordering) should be designed considering anisotropic and magnetoelastic forces. Various aspects of the theory of spin waves in rare-earth metals were considered in [59]. In the case of a ferromagnetic rare-earth metal, the simple theory must be supplemented by considering the energy contributions of the crystal field and magnetoelastic forces. The first of these contributions was taken into account in [1], in which dispersion relation for zero temperature is proposed. Anisotropy leads to the appearance of a gap in the spectrum of spin waves, the predicted value of which, for example, for dysprosium is about $24 \mathrm{~K}$. The temperature and field dependences of the spectra are usually obtained by renormalizing the coefficients and exchange integrals. Magnetoelastic forces can be considered only in two marginal approximations. In one of them, the frozen lattice approximation, it is assumed that in the case of small $k$, the lattice deformations are frozen so that they have statistically equilibrium values independently of the oscillations of magnetic moments. In another extreme case, it is assumed that for small $k$ the strains can follow the momentum oscillations. Variants of the theory differ in the number and type of terms containing magnetostriction constants. Even greater difficulties arise when building a theory of spin waves in antiferromagnetic structures. For REMs, the dispersion relations are even more complicated [59]. At present, the theory of spin waves in complex structures in the presence of many commensurate contributions to the energy of the crystal is not yet sufficiently developed for rare-earth metals to serve as a convenient model object for their verification.

In [60] it is theoretically shown that REM with different anisotropy configurations at the Neel temperature can undergo both first- and second-order phase transitions. For the hexagonal lattice in the molecular field approximation [61] a wide range of different new 
types of phase diagrams has been proposed. It was found that the Neel temperature in the presence of a strong exchange within the basal plane can split in the magnetic field into two lines of phase transformations of the first and second order, which was subsequently proved experimentally [62-64]

The dominant character of a magnetic ordering turned out to be connected with the sign of the coefficient determining the relation of the quadrupole moment of f-electrons with the direction of the total mechanical momentum J. In terbium, dysprosium, and holmium the coefficient is negative, while in erbium and thulium it is positive. This explains the fact that in the last two metals the higher order crystal field components favor the orientation of the moments parallel to the $c$-axis. At the same time, the particular arrangement of the moments depends on the relative magnitudes and signs of these components. In thulium, all crystal field effects only favor a single direction of spins parallel to the $c$-axis. In other heavy REMs, there is always a probability of finding a moment with a component outside the plane, and it increases in the series from holmium to terbium.

Spin ordering in heavy REMs is mainly of two types. First, longitudinal oscillations of the spin amplitude along the c-axis (erbium, thulium). Second, a helical structure with moments lying in the basal plane (terbium, dysprosium, holmium). More complex structures are combinations of these two structures. Although the existence of periodic spin structures is supported by long-range exchange forces, their exact form is determined by anisotropy and magnetostriction, each of which favors the ferromagnetic ordering. At lower temperatures, the energies associated with anisotropy and magnetostriction become larger and larger, and their contribution to the decrease in spin turning angles with decreasing temperature increases, hence they should be important in determining the possible transition to a collinear FM state at the lower Curie temperature. In $[59,65,66]$ it is assumed that magnetostriction is the dominant process that stabilizes the FM state. At the AFM-FM transition, the energy barrier separating the magnetically ordered states is compensated mainly by the magnetoelastic energy increasing during cooling [67,68]. Spontaneous magnetostriction decreases the thermodynamic potential of the FM phase more strongly than the AFM, which leads to a decrease in the TD potential difference and phase change. It was shown by Dzyaloshinskii [28], that the magnetostriction along the hexagonal axis $c$ in REM is due to the dependence of the exchange energy on the lattice parameter $c$. Thus, the AFM-FM transition arises not only due to changes in the energy spectrum of the conduction electrons but also due to an increase in the magnetoelastic energy of the exchange interaction when cooling below the Neel temperature.

Despite all said, the science of rare-earth magnetism is still on the threshold of understanding how the interaction of different groups of electrons leads to manifold magnetic structures and observable excitation spectra. The goal of a fully microscopic theory of lanthanide magnetism is to achieve the best possible understanding of magnetic structures, excitation spectra, and microscopic magnetic properties, expressed in terms of electronic structures of $\mathrm{f}$ - and s-electrons, as well as their interactions with each other and the crystal lattice.

\section{Results and Discussion}

\section{Magnetic Phase Diagrams of Heavy Lanthanides}

The basic physical and chemical properties of REMs are now sufficiently well studied. At the same time, a great variety of new magnetic structures and features of phase transitions make these metals irreplaceable objects for studying fundamental questions of the physics of magnetic phenomena.

Among heavy REMs, all elements except ytterbium (not a typical representative of this family) have hcp structure at room temperature with a space group $\mathrm{P}_{3} / \mathrm{mmc}$ with a sequence of layers ABAB. In the vicinity of the melting point $(1585,1629,1680$, and $1734 \mathrm{~K}$ for gadolinium, terbium, dysprosium, and holmium, respectively) these 4 metals change the crystal structure to bcc. Similar transitions in erbium and thulium are not observed. It is important to note that the melting temperatures of the metals monotonically 
increase from gadolinium to erbium with a slight decrease (by $5 \mathrm{~K}$ ) for thulium compared to erbium. At the same time, the boiling point, on the contrary, monotonically decreases except for erbium, where its boiling point slightly (by $53 \mathrm{~K}$ ) exceeds the analogous value of holmium [23].

Eleven (of 15 or 17) REMs have some form of ordered magnetic structures [69]. Heavy REMs have the most interesting properties; consequently, they are the most fully studied. Ordered states have been the main focus of most of the works performed on pure metals [62,70-80]. In the published works, however, there is a significant discrepancy in the results, because the single-crystalline samples obtained nowadays and investigated in the latest works have a much higher purity than those used in the earlier studies. Heavy REMs have been studied much more intensively than lighter ones; the magnetic properties, including those of single-crystalline samples, are now fairly well established [44,81-92].

Literature analysis shows that when cooling below room temperatures, all metals become ferromagnetic at some temperature characteristic for each metal (the Curie temperature), and all except for Gd pass into this magnetic state from the antiferromagnetic phase, which is formed directly from the paramagnetic phase. The Curie temperature represents the highest temperature at which ferromagnetism is observed in the absence of an external magnetic field. The temperature dependence of the critical field was previously studied both directly by measuring magnetization [93], and indirectly [94]. In the general case, the critical field increases almost linearly with temperature up to a maximum value in the vicinity of the Neel temperature, and then rapidly decreases when the transition to the paramagnetic state occurs.

Let us consider the behavior of each metal of the heavy REM series separately.

Gadolinium is a true ferromagnet over the entire temperature range, but it has [72] changes in the spin orientation, which was found in the magnetocrystalline anisotropy studies: in the temperature range 232-294 K the moments are parallel to the $c$-axis and below $232 \mathrm{~K}$ the angle of rotation of the moment relative to the $c$-axis increases, reaching a maximum of $75^{\circ}$ at $180 \mathrm{~K}$, then the rotation angle decreases and remains within $32^{\circ}$ at $4.2 \mathrm{~K}$. The question about the magnetic structure of gadolinium in the region of temperatures immediately below the Curie temperature is still open [28,95]. Gadolinium is the only metal among heavy REM that does not have an AFM phase and goes directly to the FM state at about $293 \mathrm{~K}$ [96]. Some evidence for the existence of the AFM phase in the very low critical field near $240 \mathrm{~K}$ was presented in [79,97], however, the results were not reproduced in other similar studies and were correlated with the presence of impurities and defects in the structure of the investigated samples. It is possible that these observations can also be associated with the anomalous behavior below $240 \mathrm{~K}$ along the easy magnetization direction [98-100]. According to neutronographic data [99], the c-axis is the easy one in the temperature range from the Curie temperature to the spin-reorientation transition temperature $232 \mathrm{~K}$ (Figure 2).

In $\mathrm{Tb}$ (Figure 3) spiral AFM structure exists in the range $222-228 \mathrm{~K}$ in a magnetic field less than 155 Oe. The critical field values of 300-800 Oe published earlier [77,101], can probably be related to the presence of impurities in the samples, which play the role of capture centers and thus prevent the destruction of the weakly bound AFM phase. Dependence of the critical field on temperature $H_{\text {crit }}(T)$ has a symmetric form with an obvious maximum at $226 \mathrm{~K}$. As the magnetic field increases in the same temperature interval in fields up to $5 \mathrm{kOe}$, a wide region of an intermediate fan phase existence is observed. It exists between the FM and PM order, that is, in the temperature range $222-227 \mathrm{~K}$ and the maximum field value of $5 \mathrm{kOe}$. In the low-field region, it is replaced by helical AFM ordering. The fan phase disappears at $227.3 \mathrm{~K}$ in the field of $\sim 5 \mathrm{kOe}$. A characteristic feature of the phase diagram presented in this paper is a rather wide region of the fan phase existence. It is possible to find only one phase diagram containing the fan-type phase [101], other authors did not even include this phase in the diagram, since its existence was probably proved only by only one experimental technique. However, the final solution of the fan existence in terbium remains open, since ab initio calculations do 
not allow calculations of fan-like structures in heavy REMs. They only show that, if one simulates a collinear AFM structure in the specified range of temperatures and magnetic fields, one can conclude that its existence is more likely along the hard magnetization axis than in the basal plane. No evidence for the existence of any collinear AFM structures could be found in terbium. Thus, detailed studies of elastic thermal neutron scattering on single-crystalline terbium are necessary to more accurately determine the type of magnetic ordering in this temperature and field range.

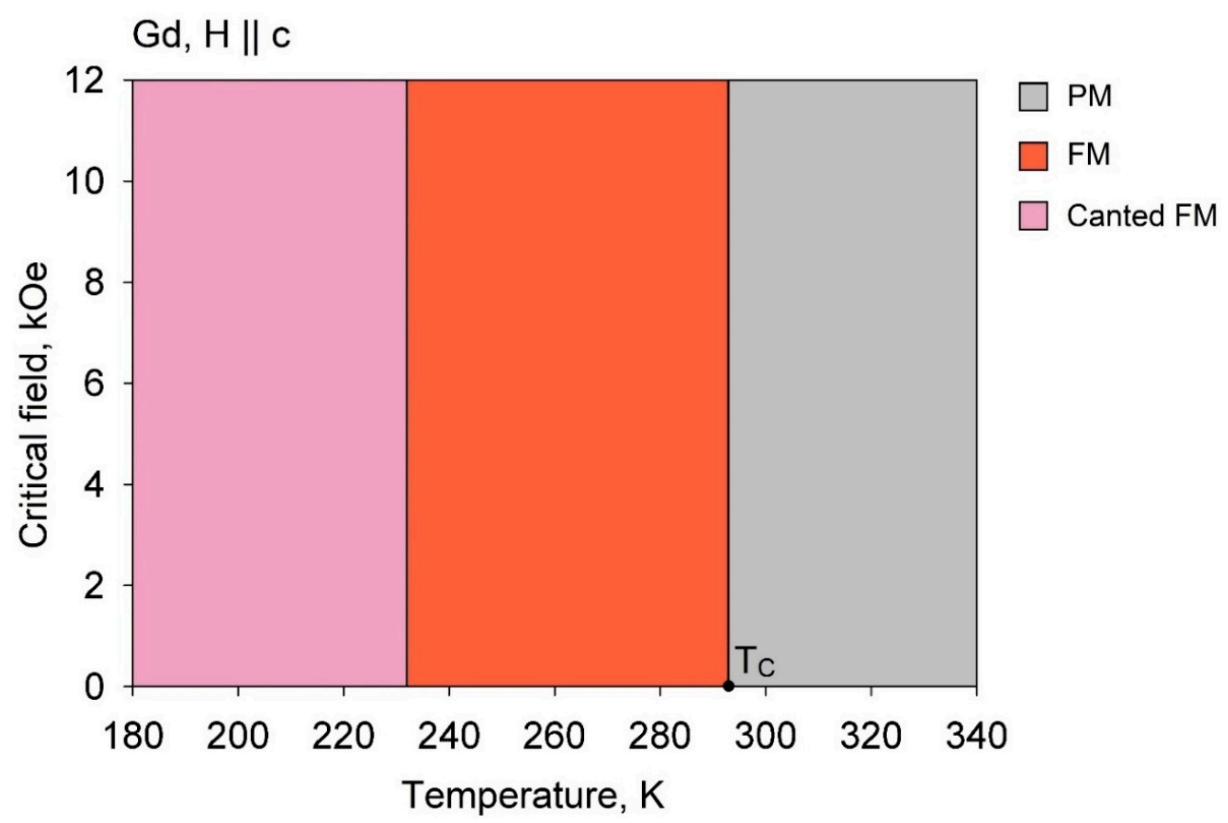

Figure 2. Magnetic phase diagram of Gd. The experimental data has been taken from [72].

In fields above $5 \mathrm{kOe}$, there are only two magnetic phases. After the fan phase is completely suppressed, the boundary between the FM and PM phases begins to slowly shift towards higher temperatures-up to $228 \mathrm{~K}$ in a $75 \mathrm{kOe}-w i t h$ increasing magnetic field, which is the typical behavior for the Curie temperature [71,102].

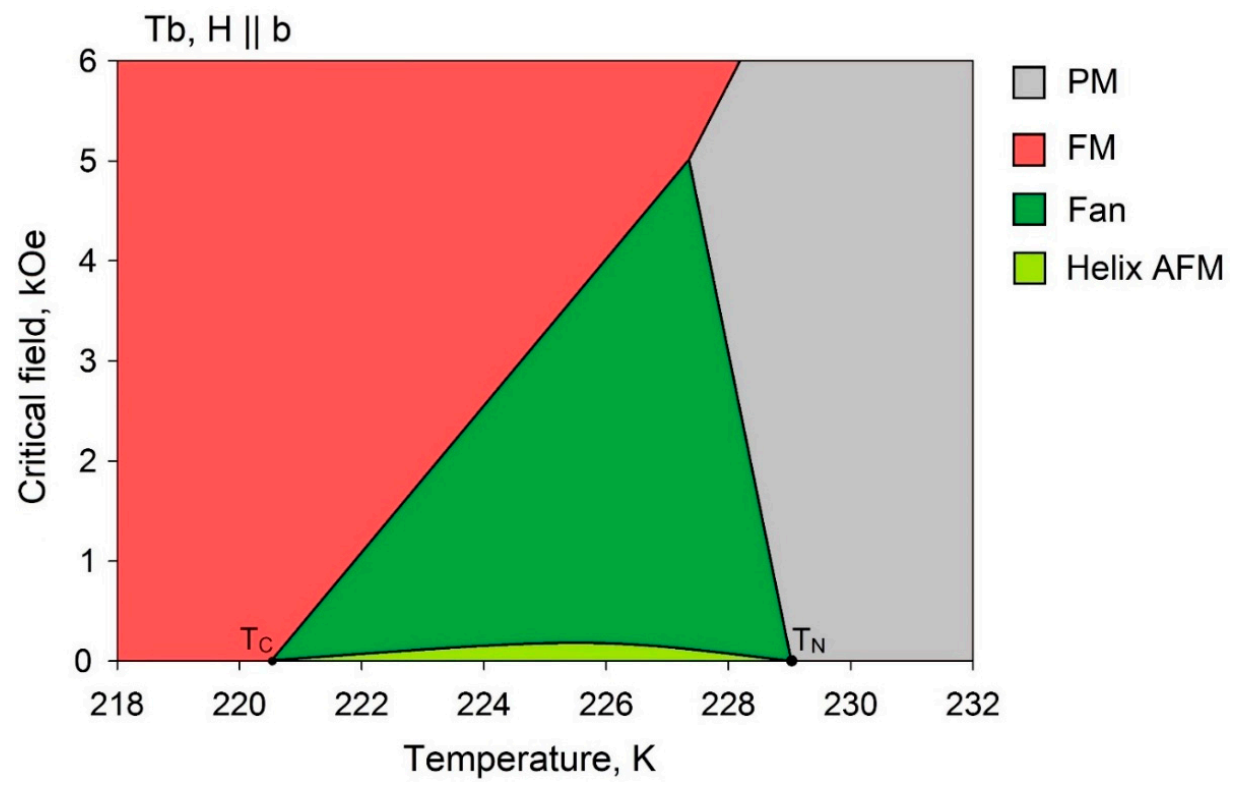

Figure 3. Magnetic phase diagram of $\mathrm{Tb}$. The experimental data has been taken from [71]. 
In dysprosium, the region of helical AFM phase existence (85-178.5 K) is much wider than in terbium (Figure 4). At a high magnetic field in dysprosium, intermediate fan phases exist at temperatures above $125 \mathrm{~K}$. The first intermediate fan phase, the existence of which has been confirmed by different experimental data of magnetization, MCE and ultrasonic measurements $[73,103,104]$ exists in the temperature range from 125 to $180 \mathrm{~K}$ in the range of magnetic fields from 5 to $23 \mathrm{kOe}$. At the same time, recent neutronographic studies and theoretical calculations $[46,105]$ indicate heterogeneity and the presence of a broader region of magnetic ordering of this type. In this case, probably, the vast region of the fan phase existence consists of areas with long and short-range order depending on the chemical purity of the sample, which in some works are interpreted as different types of fan ordering $[73,106,107]$. An extensive intermediate phase region, probably a fan with a helical structure, exists in the range $125-180 \mathrm{~K}$ and $5-25 \mathrm{kOe}$. A similar phase is observed in holmium [108], for which the existence of a helifan structure was theoretically proved. The specific boundaries of the helifan-type phase existence require clarification by neutronography. In addition, a second intermediate phase in the region of $180 \mathrm{~K}$ in the field range of 6-23 kOe is observed in dysprosium. A similar result was previously published in [88], which suggested the existence of a vortex magnetic structure in this region, but it found no further experimental confirmation, although the existence of such unstable structures had previously been predicted theoretically [109] in the case of a magnetic field applied along the hard magnetization $c$-axis. Because the vortex magnetic ordering is no longer found in any of the heavy REMs, it is reasonable to assume the existence of another intermediate fan phase in this region, which is indirectly confirmed by the results of neutronography [105].

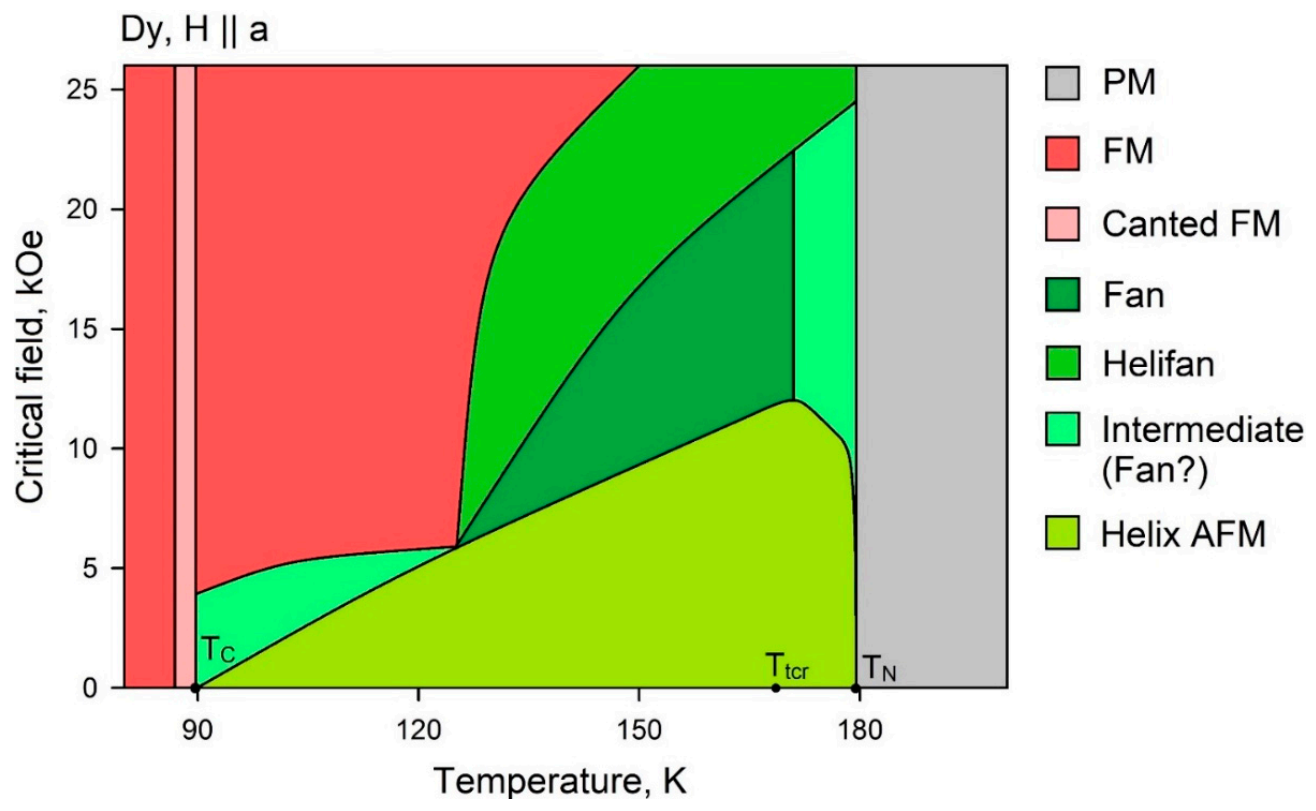

Figure 4. Magnetic phase diagram of Dy. The experimental data has been taken from $[46,73,105]$.

The phase detected in the temperature range from 90 to $130 \mathrm{~K}$ is probably intermediate between FM ordering and fan phase with long-range ordering. Likely, it exhibits predominantly FM ordering with portions of fan phase with short-range ordering. [46,105]

In the Curie temperature region, according to theoretical calculations [46], there is a canted FM ordering. The existence of such a phase has not yet been established experimentally, and the reason for its occurrence may be a combined magnetic-structural phase transition in dysprosium near $90 \mathrm{~K}$, which causes local distortion of the FM structure. At the same time, the region of such a phase existence is very limited; in lower temperatures, the common collinear FM ordering is observed. 
In the region of temperatures above the Neel temperature $(179 \mathrm{~K})$ dysprosium is paramagnetic. Some works suggest the presence of antiferromagnetic clusters in the PM region [73,110], but these assumptions require experimental verification.

Holmium is ferromagnetic below the Curie temperature $\left(\mathrm{T}_{\mathrm{C}}=20 \mathrm{~K}\right)$ and antiferromagnetic between the Curie and the Neel temperature $\left(T_{N}=131 \mathrm{~K}\right)$ in the range of magnetic fields from 0 to $\sim 30 \mathrm{kOe}$ (Figure 5). In fields up to $2 \mathrm{kOe}$ at low temperatures $(13 \div 20 \mathrm{~K})$ a spiral ordering of FM type (cone) with the cone axis directed along the hard magnetization $c$-axis is observed. At temperatures below $50 \mathrm{~K}$ magnetic satellites of the 5 th and 7 th orders are observed, which indicates a strong deviation of the magnetic moments in the direction of the easy magnetization axis in the basal plane [111]. Application of the field in the basal plane leads to the transformation of the conic ordering into an FM structure [112]. Anomalies were also found to represent transitions into intermediate phases of the fan type with helicoid structure (helifan) and "simple" fan (as the magnetic field increases) as the AFM phase transforms into ferromagnetic phase in the temperature range from 45 to $95 \mathrm{~K}$. According to theoretical estimations, the existence of helifan type $3 / 2$ is recognized as the most probable in holmium, however, neutronographic studies are required to accurately determine its type.

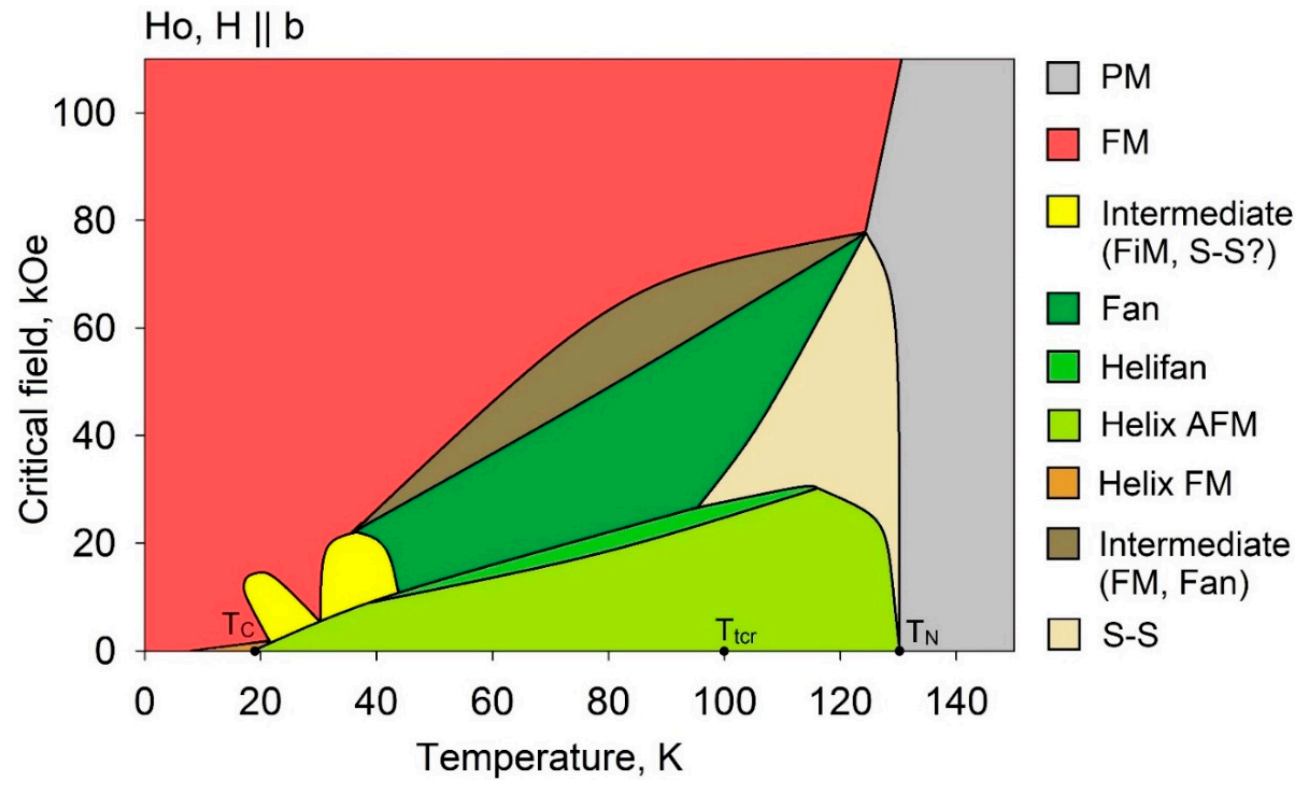

Figure 5. Magnetic phase diagram of Ho. The experimental data has been taken from [62].

A further field increase causes the fan structure to collapse and the transition to full FM ordering [111,112]. In holmium, there is an intermediate region of coexistence of the two phases between the fan and FM phases. In comparison with the five intermediate phases observed in [113], detected from magnetoresistance measurements, only one phase is observed in the present work, and it can be assumed that, despite the high purity of the crystals used in the present work, impurity effects still play a significant role in those regions of the phase diagram that are most sensitive to the magnetic field (intermediate metastable phases). These impurities can be the cause of mixed-phase states, such as "ferro+fan" which combines features of both fan and FM structures.

Two regions of spin-slip structures existence are observed between 20 and $35 \mathrm{~K}$ (I) and between 35 and $42 \mathrm{~K}$ (II). The extensive region in the phase diagram between $\sim 95 \mathrm{~K}$ and the Neel temperature in the field range from 30 to $80 \mathrm{kOe}$ is associated with the presence of a spin-slip structure (III), an indication of which was observed from magnetic measurements at $98 \mathrm{~K}$ in [84]. The existence of such structures has previously been predicted [114,115], but so far there has been no experimental confirmation of these structures' existence using a set of different experimental techniques. The spin-slip model explains the transitions 
in magnetic helices in REM in terms of simple commensurable structures. The spin-slip theory is based on experimentally observed localized spin defects due to, among other things, the purity of the samples [116]. Therefore, the number of varieties of such spin configurations can be extremely large. In [117] it has been suggested that the appearance of spin-slip type defects in a simple (antiferromagnetic) helix can lead to various kinds of spontaneous ferrimagnetic structures (in the limiting case) with macroscopic momentum, which appears to be observed in thulium [106,118-120].

Outside the temperature range of $19-128 \mathrm{~K}$ and the field range of 10 to $80 \mathrm{kOe}$ holmium is a typical ferromagnet. At temperatures above $131.7 \mathrm{~K}$ holmium is paramagnetic (Figure 5).

The most complex magnetic structures are observed in erbium (Figure 6). Below $20 \mathrm{~K}$ erbium has a ferromagnetic helix (cone) type structure, which in higher fields consistently transfers to a fan and then to a collinear FM phase. The value of the Curie temperature according to different sources varies in the range from 16 to $20 \mathrm{~K}$, which, apparently, is associated with the purity of the studied samples. [121,122] Erbium, like all heavy REMs, has an extensive AFM ordering region in the range of 20-85 K. The subregions of the long-range magnetic order are also distinguished in this region. In the range of $20-50 \mathrm{~K}$, both transverse and longitudinal components of the magnetic moment oscillate, i.e., a complex spiral is realized. In this temperature range, spin-slip defects of various types and, in the limit, ferrimagnetic ordering are likely to occur. This region is intermediate: a helicoidal component of the magnetic moment in the basal plane exists here. In the interval 52-84 K, a longitudinal spin-wave is observed in erbium, which is characterized by the oscillation of the longitudinal component of the magnetic moment along the caxis $[57,123,124]$. The spin-wave is a limiting case of the helical structure. Below $52 \mathrm{~K}$, this wave is supplemented by oscillations of the magnetic moment components in the basal plane of the helix type, with the period of the helicoid coinciding with the period of the longitudinal spin-wave. A whole cascade of transitions between spin-slip phases of various configurations exists in erbium in the temperature range $40-70 \mathrm{~K}$ [125]. In the same temperature range, an additional transformation of the magnetic structure associated with the transition of erbium to a commensurate phase with wave vector $6 / 23$ is observed. Apparently, the coexistence of phases with different wave vectors of the magnetic structure is observed in this temperature range. The temperature boundary of this effect (in the region of $40 \mathrm{~K}$ ) is determined by the temperature region of a significant increase in magnetic anisotropy, sufficient to suppress the effect of commensurability [55]. In erbium, the presence of certain phase transitions depends particularly on the purity of the samples: thus, when in [126,127] such commensurate points were found as $2 / 7,3 / 11,4 / 15,5 / 19$, $6 / 23, \frac{1}{4}$ и5 $/ 21$, then in [63] 5/19, $\frac{1}{4}$ и5 $/ 21$ are only found.

Above the AFM ordering boundary, there is a narrow region of phases with fantype helicoidal ordering (helifan), as well as a number of intermediate phases containing features of cone, fan, and ferromagnetic ordering. The number of commensurate structures in erbium does not exceed 7-8 [126,128]. Impurity atoms can form a so-called impurity magnetic center and distort the magnetic structure in their environment $[129,130]$. Applying or removing the field leads to irreversible changes in the local magnetic moment on the impurity atom (using tin atoms as an example).

The magnitude of the cone half-dissolution angle $\Theta$ in erbium is related to the structural arrangement of the AFM structure. Exposure to a magnetic field can induce a series of consecutive spin-slip structures in the basal plane. The mechanism of this relationship can be explained as follows. Magnetizing of the crystal along the $c$-axis leads to a magnetostrictive change in the initial sample volume and the lattice parameter $\mathbf{c}$. This violates the commensurability conditions of a given magnetic structure and can lead to a transition to a structure with a different wave vector. The resulting violation of the initial balance of crystal field energy, exchange, and magnetoelasticity causes a jump change in the $\Theta$ angle value. An explanation related to the influence of the magnetic field along the $c$-axis on the magnetic structure in the basal plane, determined by the competition of interplanar 
exchange and sixth-order anisotropy, is also possible. The transformation of the initial structure when the cone half-solution angle changes are related to the different dependence of the exchange $\left(\sim \sin ^{2} \Theta\right)$ and sixth-order anisotropy $\left(\sim \sin ^{6} \Theta\right)$ on this angle [131]. This explanation is also true in the case of holmium.
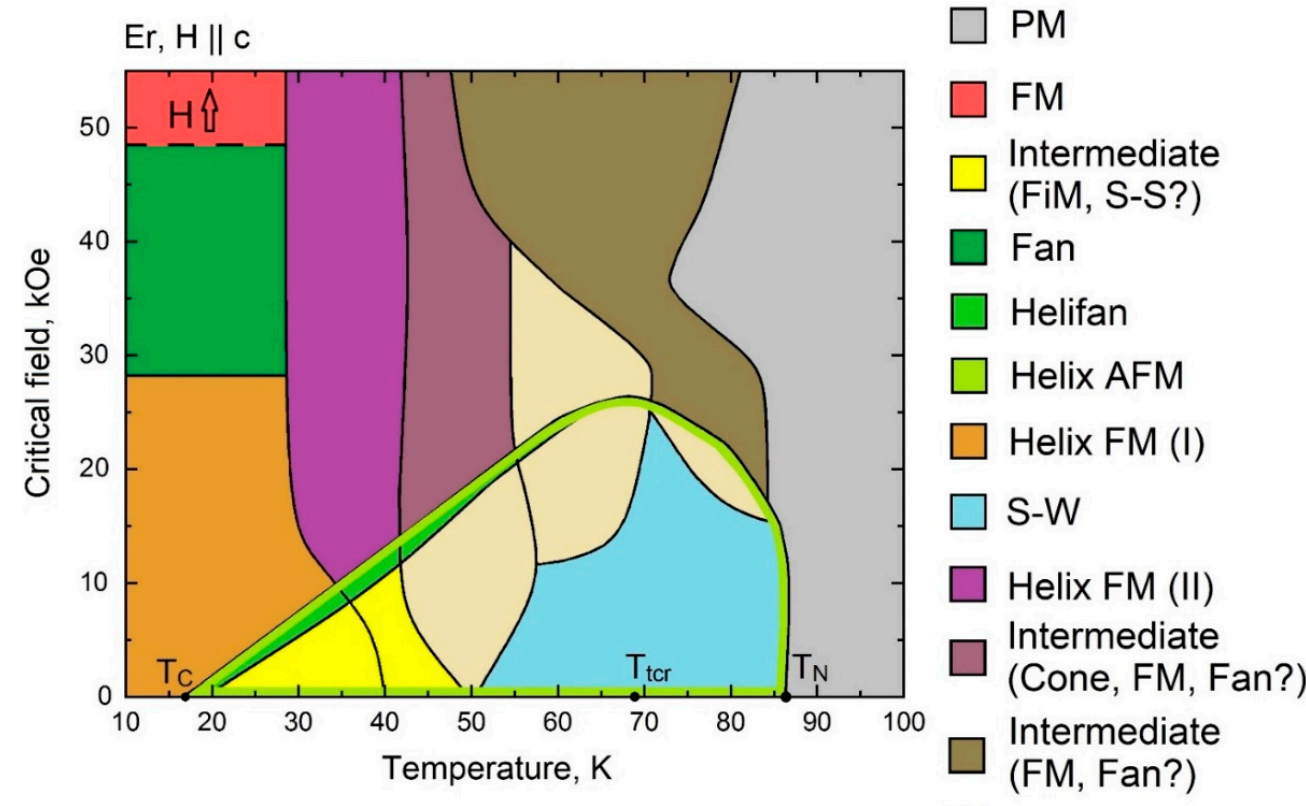

S-S

Figure 6. Magnetic phase diagram of Er. The experimental data has been taken from [64].

Thulium (Figure 7) also has an extensive AFM ordering region in the range from zero to the Neel temperature $(56 \mathrm{~K})$. In the region of low temperatures and up to $32 \mathrm{~K}$, the metal has an antiphase domain structure in which the magnetic moments of the four atomic layers lying in the hcp basal plane are oriented upward, while the magnetic moments of the three closest layers are oriented downward. Thus, there is no variety of spin-slip phases in Tm: they are all transformed into a ferrimagnetic structure that is commensurate with the crystal lattice. As the temperature increases, the antiphase structure begins to break down due to the appearance of the FM component, and in the range of 32-40 K, the magnetic structure is incommensurate with the crystal structure. This intermediate phase contains features of both low-temperature ferrimagnetic ordering and the static longitudinal spin-wave, which is observed in thulium up to $56 \mathrm{~K}$. A number of authors did not observe this intermediate phase in Tm at all [132], which seems to be related to the purity of the material. The axis of the magnetic moment oscillations in the spin-wave is the hexagonal $c$-axis.

The peculiarities of the magnetic behavior of erbium and thulium, as compared to terbium, dysprosium, and holmium, is that in these metals the magnetic anisotropy tends to orient the magnetic moments along the $c$-axis. As a result, in Tm, in the FM region below $32 \mathrm{~K}$, the $c$-axis is the easy one, while in erbium it is the axis of the FM helix below $20 \mathrm{~K}$. Between the Curie and Neel temperatures, the $c$-axis in these metals is the spin-wave modulation axis. If the field is applied along the $c$-axis, there is a gradual collapsing of the FM helix (cone) in erbium, which continues in fields above 15 T. [133] As a rule, the magnetic moments are finally oriented parallel to each other in fields above $15 \mathrm{~T}$ : in fields above $17 \mathrm{~T}$ at helium temperatures, a ferromagnetic structure is realized in erbium [133]. 


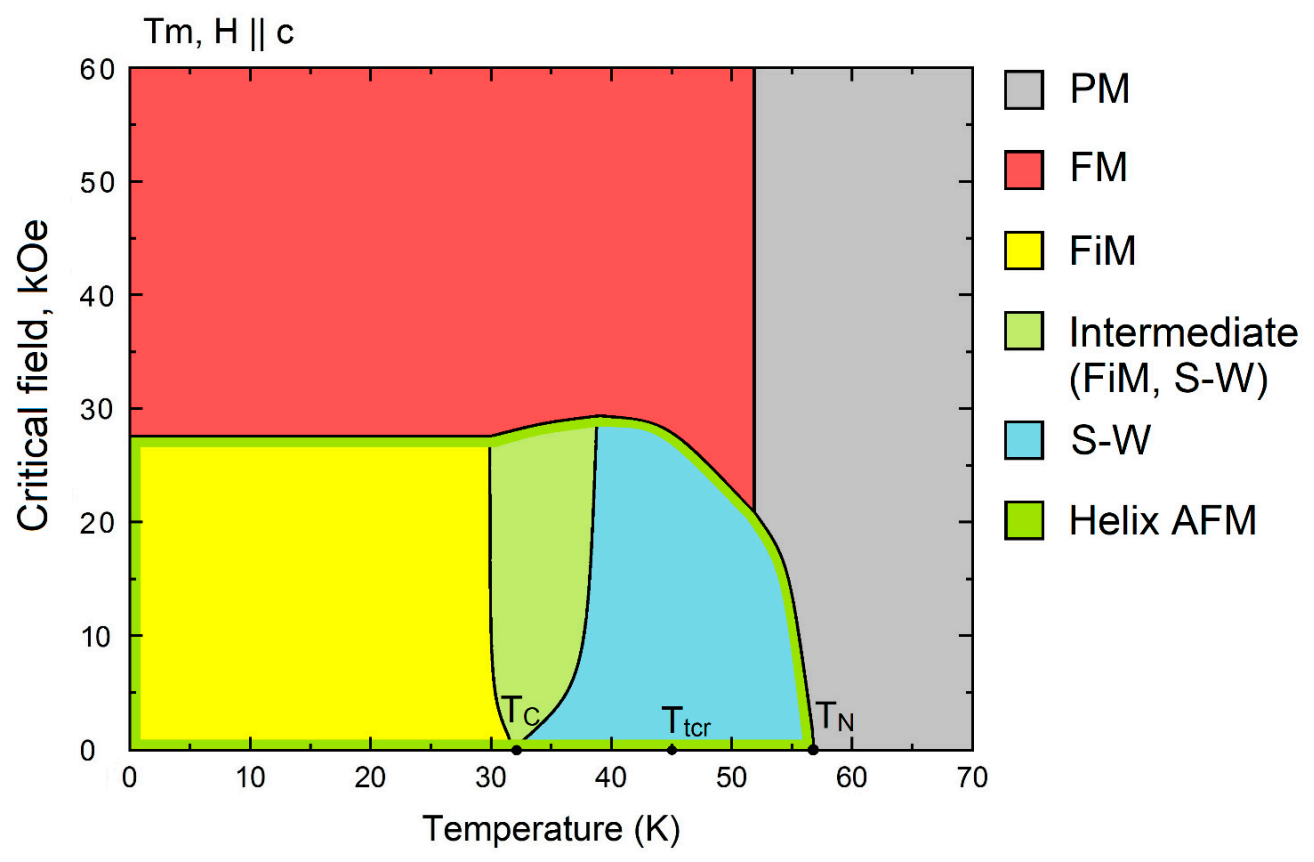

Figure 7. Magnetic phase diagram of Tm. The experimental data has been taken from [118].

The analysis of magnetic phase diagrams allows us, despite the above-mentioned differences between the individual elements, to highlight a number of common properties. First of all, this is the uniformity of the crystal structure (hcp). The interatomic distances in all metals are fairly constant, although the ionic radii noticeably decrease with the order number of the element due to lanthanide compression. In addition, in all heavy REM FM and AFM spiral phases coexist (except for gadolinium). The main types of helical structures are presented in [55]. The period of modulated structures usually changes with temperature, taking incommensurable or commensurable values with respect to the period of the crystal lattice. Anisotropy in the basal plane can cause a significant transformation of a simple helical structure and lead to "locking" of the wave vector during the phase transition from incommensurable to commensurable structure. Increasing anisotropy leads to "binding" of individual spins into "bunches" and, in the limit, to a complete collapse of moments to one of the easy directions. The ideal spiral structure, characterized by a constant angle of moment rotation, is a very crude approximation. A more accurate approximation is a model in which the angle is modulated as it moves along the $c$-axis, not randomly, but in a well-defined way. The energies of these phases are close, since the critical fields in which the collapse of the helical structure are only a few percent of the effective exchange fields.

In five of the considered heavy REMs, different types of ferromagnetic spin ordering were found. In terbium and dysprosium, there is a parallel orientation of moments in the basal plane in the direction of light magnetization. In holmium and erbium, no complete ordering of moments is observed, and the structure has a ferromagnetic helical arrangement (ferromagnetic cone). One of the momentum components is directed parallel to the $c$-axis and has a ferromagnetic ordering, while the helical configuration is preserved in the basal plane. For the same reasons, in [134] it was proposed to introduce a weak axial component (along the $c$-axis) for dysprosium. The appearance of this component was observed at temperatures below $100 \mathrm{~K}$ (with poor repeatability on three samples); accordingly, the appearance or disappearance of this component may be related to the presence of impurities.

The character of phase transitions is significantly affected by the magnetic field, pressure, defects, and a number of other factors [135-138]. First-order magnetic phase transitions (FOPT) take place between phases of any symmetry and, in addition to hysteresis phenomena, are usually accompanied by metastable phenomena and the appearance of 
various intermediate phases. Neutronographic measurements [139-142] showed that, for example, in holmium, there is a much stronger tendency to spiral ordering than in terbium. Spin waves in erbium and thulium reveal odd higher harmonics of the wave vector as the temperature decreases, corresponding to a symmetric transformation of the sinusoidal structure into an antiphase one. In the framework of the molecular field model it was shown [45], that during the development of a spin wave-type structure, the amplitude of the sinusoidally varying momentum reaches the value inherent in a free ion at $T \approx 0.5 T_{N}$. Since this value of momentum is maximum, a further increase in energy can be achieved by the development of higher harmonics (conversion of the sinusoid into a periodic sequence of rectangular form, which is observed experimentally).

In the present work, the values of the tricritical temperatures were theoretically calculated for all heavy REMs for the first time. The magnetization curves of heavy REM usually have a maximum at the para-antiferromagnetic transition (the Neel temperature), which is accompanied at lower temperatures by a very sharp increase in magnetization when the ferromagnetic phase is formed, usually through intermediate metastable phases. Because of this general behavior of magnetization, it is correct to apply the relations for determining the critical temperatures obtained in [2]. The results are presented in Table 1.

Table 1. Theoretical values of the tricritical temperatures of heavy REM.

\begin{tabular}{|c|c|c|c|}
\hline Metal & $\begin{array}{c}\text { The Neel } \\
\text { Temperature, } \mathrm{T}_{\mathrm{N}}, \mathrm{K}\end{array}$ & $\begin{array}{c}\text { The Paramagnetic Curie } \\
\text { Temperature, } \Theta_{\mathbf{P}}, \mathbf{K}[23,33,133]\end{array}$ & $\begin{array}{c}\text { Tricritical } \\
\text { Temperature, } \mathrm{T}_{\mathrm{tcr}}, \mathrm{K}\end{array}$ \\
\hline $\mathrm{Tb}$ & 229 & $239, \perp c$ & 236 \\
\hline Dy & 179 & $164, \perp c$ & 168 \\
\hline Ho & 130 & $88, \perp c$ & 100 \\
\hline Er & 86 & 61.7, \|c & 68 \\
\hline $\mathrm{Tm}$ & 56.5 & $41, \| \mathrm{c}$ & 45 \\
\hline
\end{tabular}

In the case of REM, the critical temperature is the point at which the difference between the PM and FM ordering disappears, that is, the boundary of the FOPT transforms into the boundary of SOPT. By definition, $\frac{d H_{c r}}{d T}=0$ at $T=T_{c r}$. For the existence of a tricritical point, the simultaneous existence of three phases is necessary, and the condition that the derivative of the critical parameter is equal to zero in temperature is not always satisfied (a typical example is the triple point of water). The variety of magnetic phase transitions in heavy REM does not exclude the existence of tricritical points in phase diagrams. Moreover, a case of comparison of experimental and theoretical values of the tricritical temperature for dysprosium was found in [73]. It is pointed out that the theoretical value of $168.4 \mathrm{~K}$ obtained by the authors is lower than the experimental value of $172 \mathrm{~K}$ obtained in the same paper, but is slightly higher than the $165 \mathrm{~K}$ obtained in [143] on samples of the worst quality. At the same time, in [73] the experimental value of the tricritical temperature is determined indirectly, by the type of phase boundaries in the magnetic phase diagram. The accuracy of this method is insufficient, so the experimental determination of tricritical temperature values requires the explicit detection of three phases with a physically distinguishable phase boundary. The theoretical value of the terbium tricritical point does not correspond to the definition of the tricritical point, since at $236 \mathrm{~K}$ either the transition from FM to PM phase or a completely paramagnetic state is observed in $\mathrm{Tb}$. It can be noted that in all the rest heavy REM the tricritical point is more likely to be in the region of helical AFM ordering or on its boundary with other phases. As in terbium, this region is the narrowest in the series of heavy REMs, the experimental detection of the tricritical point in terbium is of particular interest and difficulty.

The PM-APM transition in heavy REM has the features of both the first- and secondorder phase transitions. Within the molecular field model, the following equation for the transition temperature from the paramagnetic to the antiferromagnetic state can be derived [45]: 


$$
k T_{N}=\frac{2}{3} \mathcal{J}\left(\vec{q}_{m}\right) J(J+1)=\frac{2}{3} \dot{j}\left(\vec{q}_{m}\right)\left(g_{J}-1\right)^{2} J(J+1)
$$

This formula is valid for any antiferromagnetic structures. If one assumes that the elementary exchange $\dot{j}\left(\vec{q}_{m}\right)$ is constant for the entire series of heavy REMs, then one can conclude that there is a linear dependence of the Neel temperature $T_{N}$ on de Gennes function $G=\left(g_{j}-1\right)^{2} J(J+1)$-is the average value of the spin projection on the total mechanical momentum. However, due to the limitations of the molecular field model, the best agreement with the experimental data is observed for the ratio $T_{N} \sim G^{2 / 3}$ [144]. In the present study, the validity of this relation was experimentally proved for high-purity single crystals of heavy REM (Figure 8). This dependence was first proposed in [145] but tested only in the case of alloys $[144,146,147]$.

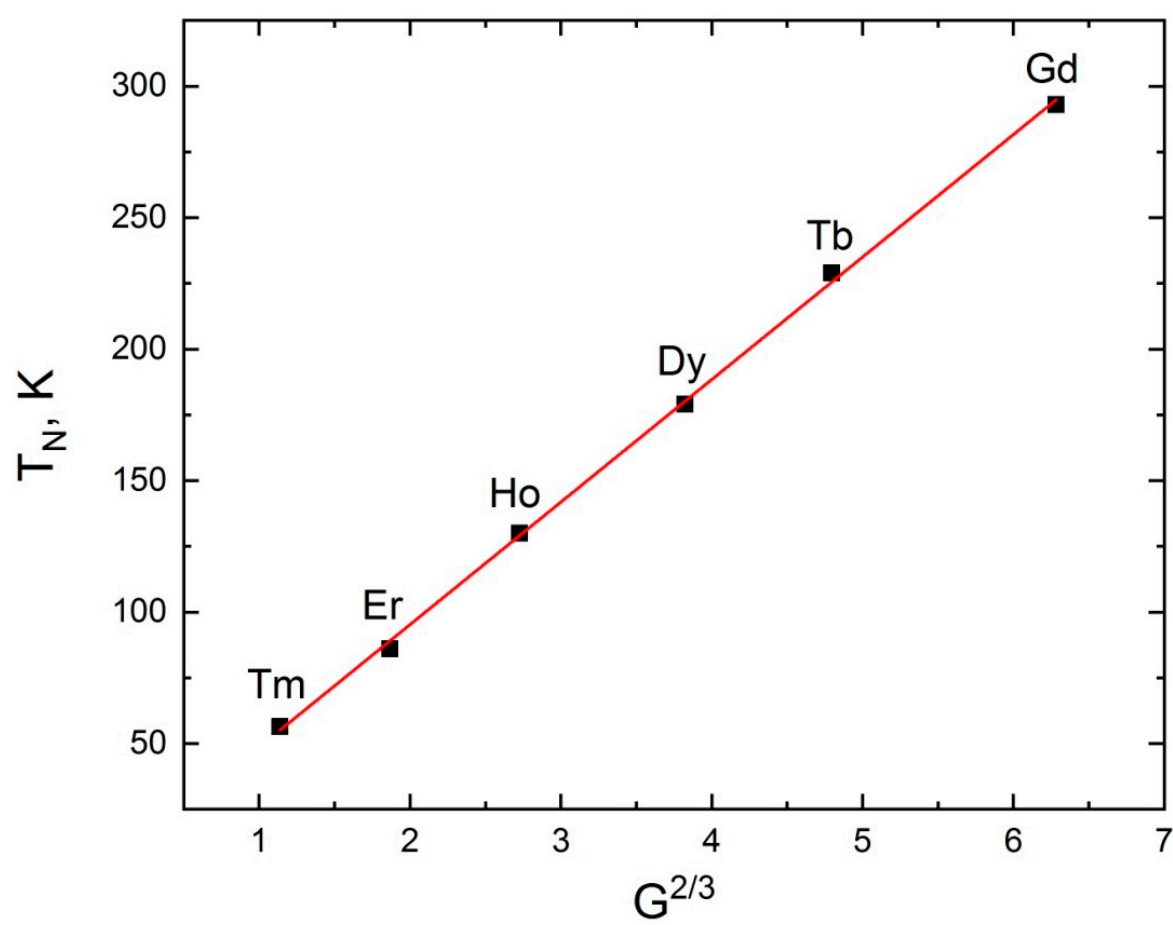

Figure 8. The Neel temperature of heavy lanthanides dependence on de Gennes function.

We can conclude that in heavy REM single crystals there is a one-to-one functional dependence between the AFM-PM transition temperature and de Genne's function. This fact can be explained by the character of the temperature dependence of the spin rotation angle (the angle between the magnetic moments of the neighboring magnetic layers) in the helical antiferromagnetic structure on de Genne's function. The value of the rotation angle weakly depends on the temperature at small values of the function, with increasing $G$ the change becomes more noticeable, at a certain value the rotation angle decreases sharply to zero at some finite temperature.

At the same time in [84], it is found that the Neel temperature in holmium is split by the magnetic field (similarly, in erbium). This result confirms the effect [135], observed in REMs with a parameter of the order of 4 (holmium, dysprosium, terbium), occurrence in the field.

The diversity and complexity of magnetic phase transitions in heavy REM require a unified theory of mixed magnetic phase transitions, by analogy with the Bean-Rodbell and Kittel models for FOPT [148-150] and Landau theory [151] for second-order phase transitions (SOPT). Thus it is reasonable to find a phenomenological parameter that would serve as an indicator of phase transformation in heavy REM. In the case of REM, taking into account the phase transition cascade, which is observed in most of the considered metals, 
it is probably not quite correct to speak about a specific phase transition temperature, but rather about the region of material phase transformation depending on the magnetic field.

In this sense, the magnetic contribution to the heat capacity $C_{m a g n}(T, H)$ can be interpreted as the order parameter in the theory of first- and second-order magnetic phase transformations in non-zero fields. Figure 9 summarizes the displacements of the maximum in the magnetic specific heat capacity of heavy REM, where $T / T_{p k}$ is the ratio of the measured temperature to the temperature in which the maximum of the magnetic specific heat at the certain magnetic field is observed. The experimental data are compared with the theoretical calculations (solid black line) taken from Figure 9 of Ref. [152]. One can see that the common trend is observed for all metals; the critical field value is observed, i.e., the value where the maximum begins to shift towards higher temperatures. The following values are experimentally observed: Gd-1.8 T; Tb-0.3 T; Dy-0.3 T; Ho-2 T; Er-2.3 T, comparing with a theoretical value of $0.4 \mathrm{~T}$ from [152].

Thus, it can be apparently seen that the field-induced non-monotonic behavior of the maximum in the magnetic heat capacity is an effect common to all of them. Obviously, the appropriate mathematical apparatus of such a theory needs to be elaborated.

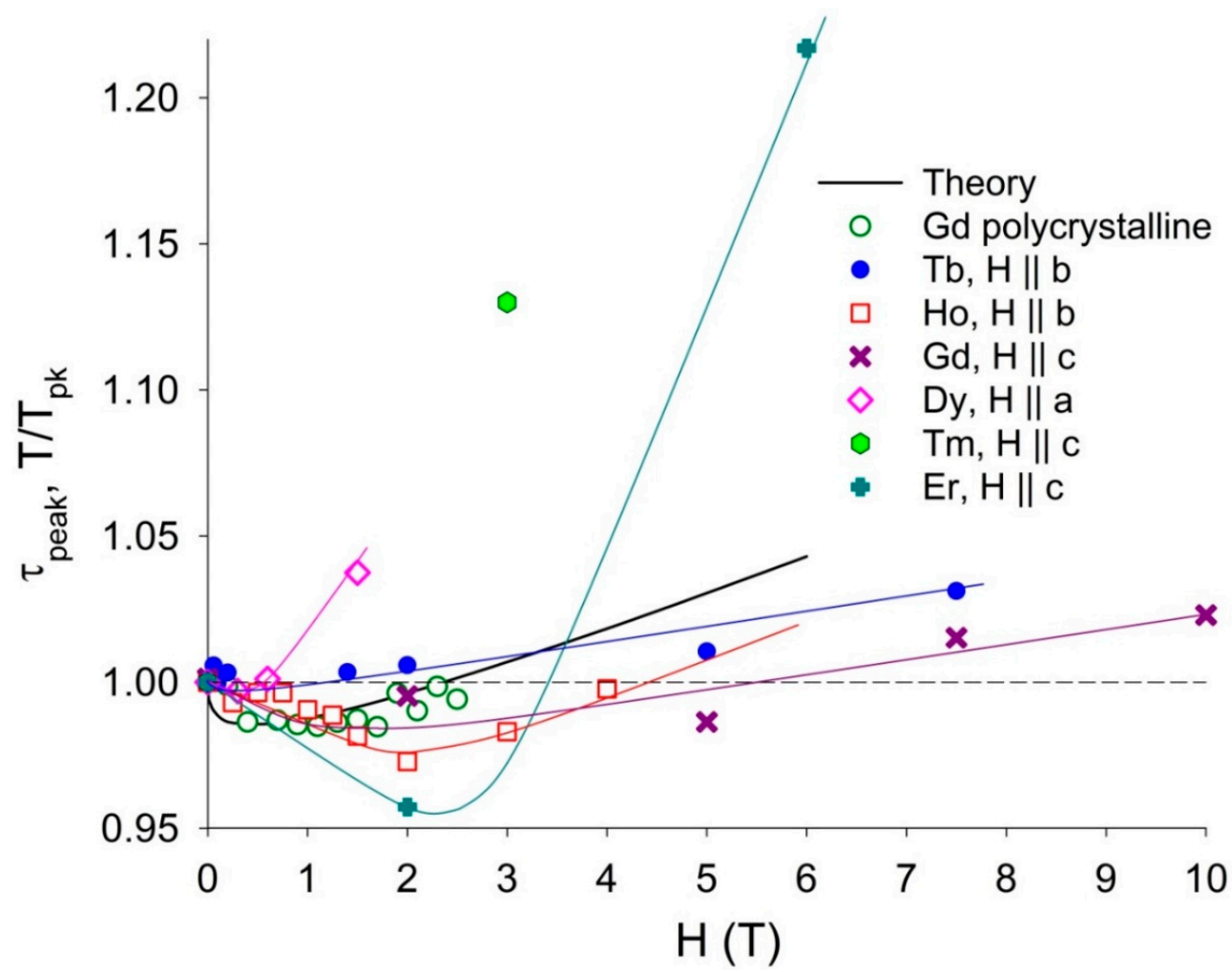

Figure 9. Position of the maximum in the magnetic specific heat of heavy REM in dependence on the magnetic field. The experimental data for Gd is taken from [72,153], Tb [71], Dy [46,73], Ho [62], Er [154], Tm [155]. Lines drawn through the data points are guides for the eye. The theoretical curve (solid black line) is from [152].

\section{Conclusions}

For the first time, the densities of states (DOS) of heavy REMs were calculated using the projector-augmented waves method (PAW). DOS for pure metals do not differ significantly, except for gadolinium. This fact distinguishes it from the series of heavy REM (absence of antiferromagnetic ordering), while at the same time explaining the general laws of the existence of magnetic phases in heavy lanthanides. From this point of view, for the first time the last published magnetic phase diagrams of metals from gadolinium to thulium, three of which were obtained by the authors of this paper, are analyzed together. In low fields, hexagonal anisotropy provides a ferromagnetic ordering of spins; as the field increases, a 
stable helical antiferromagnetic ordering is observed. Deformation of the spiral results in a fan phase with a helical structure (helifan). In high magnetic fields bulk heavy lanthanides are predominantly in fan or ferromagnetic states. The spin slip type phases arise due to the dominance of anisotropy energy over exchange energy, the existence of structural and chemical defects. For all elements, there must be a theoretical tricritical point (the point of coexistence of the three magnetic phases), the experimental detection of which remains outside the scope of this paper and requires a separate study. In this case, the theoretical value of the tricritical temperature monotonically decreases from terbium to thulium. The linear dependence of the Neel temperature of high-purity bulk heavy REMs on de Gennes function $\mathrm{G}^{2 / 3}$ was shown for the first time. In all heavy rare earth metals, there is a common, triggered by an external magnetic field, non-monotonic response of the maximum magnetic heat capacity with a minimum of the maximum temperature shift dependence at some value of the critical field, different for each element. The magnetic heat capacity can be considered as an order parameter in developing a theory of phase transitions in a magnetic field.

Author Contributions: Conceptualization, V.I.Z. and R.R.G.; methodology, V.I.Z.; software, A.S.D.; validation, A.S.D. and V.I.Z.; investigation, A.S.K.; resources, B.B.K.; writing-original draft preparation, R.R.G. and A.S.K.; writing-review and editing, B.B.K. and V.I.Z.; visualization, R.R.G.; supervision, V.I.Z. All authors have read and agreed to the published version of the manuscript.

Funding: This research received no external funding.

Institutional Review Board Statement: Not applicable.

Informed Consent Statement: Not applicable.

Data Availability Statement: The data presented in this study are available on request from the corresponding author.

Conflicts of Interest: The authors declare no conflict of interest.

\section{References}

1. Miwa, H.; Yosida, K. Magnetic Ordering in the Rare-Earth Metals with More than Half-Filled 4f Shells. Prog. Theor. Phys. 1961, 26, 693-721. [CrossRef]

2. Kitano, Y.; Nagamiya, T. Magnetization Process of a Screw Spin System. II. Prog. Theor. Phys. 1964, 31, 1-43. [CrossRef]

3. Nagamiya, T.; Nagata, K.; Kitano, Y. Magnetization Process of a Screw Spin System. Prog. Theor. Phys. 1962, $27,1253-1271$. [CrossRef]

4. Cooper, B.R.; Elliott, R.J.; Nettel, S.J.; Suhl, H. Theory of Magnetic Resonance in the Heavy Rare-Earth Metals. Phys. Rev. 1962, 127, 57-68. [CrossRef]

5. Kaplan, T.A. Some Effects of Anisotropy on Spiral Spin-Configurations with Application to Rare-Earth Metals. Phys. Rev. 1961, 124, 329-339. [CrossRef]

6. Yosida, K.; Watabe, A. Fermi Surfaces and Spin Structures in Heavy Rare-Earth Metals. Prog. Theor. Phys. 1962, $28,361-370$. [CrossRef]

7. Hughes, I.D.; Däne, M.; Ernst, A.; Hergert, W.; Lüders, M.; Poulter, J.; Staunton, J.B.; Svane, A.; Szotek, Z.; Temmerman, W.M. Lanthanide Contraction and Magnetism in the Heavy Rare Earth Elements. Nature 2007, 446, 650-653. [CrossRef]

8. Döbrich, K.M.; Bostwick, A.; McChesney, J.L.; Rossnagel, K.; Rotenberg, E.; Kaindl, G. Fermi-Surface Topology and Helical Antiferromagnetism in Heavy Lanthanide Metals. Phys. Rev. Lett. 2010, 104, 246401. [CrossRef]

9. Locht, I.L.M.; Kvashnin, Y.O.; Rodrigues, D.C.M.; Pereiro, M.; Bergman, A.; Bergqvist, L.; Lichtenstein, A.I.; Katsnelson, M.I.; Delin, A.; Klautau, A.B.; et al. Standard Model of the Rare Earths Analyzed from the Hubbard I Approximation. Phys. Rev. B 2016, 94, 085137. [CrossRef]

10. Mendive-Tapia, E.; Staunton, J.B. Theory of Magnetic Ordering in the Heavy Rare Earths: Ab Initio Electronic Origin of Pair- and Four-Spin Interactions. Phys. Rev. Lett. 2017, 118, 197202. [CrossRef]

11. Mendive-Tapia, E.; Staunton, J.B. Caloric Effects around Phase Transitions in Magnetic Materials Described by Ab Initio Theory: The Electronic Glue and Fluctuating Local Moments. J. Appl. Phys. 2020, 127, 113903. [CrossRef]

12. Tapia, E.M. Ab Initio Theory of Magnetic Ordering: Electronic Origin of Pair-and Multi-Spin Interactions; Springer Theses; Springer International Publishing: Berlin/Heidelberg, Germany, 2020; ISBN 978-3-030-37237-8.

13. Spedding, F.H.; Hanak, J.J.; Daane, A.H. High Temperature Allotropy and Thermal Expansion of the Rare-Earth Metals. J. Less Common Met. 1961, 3, 110-124. [CrossRef] 
14. Spedding, F.H.; Daane, A.H.; Herrmann, K.W. The Crystal Structures and Lattice Parameters of High-Purity Scandium, Yttrium and the Rare Earth Metals. Acta Crystallogr. 1956, 9, 559-563. [CrossRef]

15. Banister, J.R.; Legvold, S.; Spedding, F.H. Structure of Gd, Dy, and Er at Low Temperatures. Phys. Rev. 1954, 94, 1140-1142. [CrossRef]

16. Barson, F.; Legvold, S.; Spedding, F.H. Thermal Expansion of Rare Earth Metals. Phys. Rev. 1957, 105, 418-424. [CrossRef]

17. Behrendt, D.R.; Legvold, S.; Spedding, F.H. Magnetic Properties of Dysprosium Single Crystals. Phys. Rev. 1958, $109,1544-1547$. [CrossRef]

18. Elliott, J.F.; Legvold, S.; Spedding, F.H. Some Magnetic Properties of Gadolinium Metal. Phys. Rev. 1953, 91, 28-30. [CrossRef]

19. Gerstein, B.C.; Griffel, M.; Jennings, L.D.; Miller, R.E.; Skochdopole, R.E.; Spedding, F.H. Heat Capacity of Holmium from 15 to $300^{\circ}$ K. J. Chem. Phys. 1957, 27, 394-399. [CrossRef]

20. Green, R.W.; Legvold, S.; Spedding, F.H. Magnetization and Electrical Resistivity of Erbium Single Crystals. Phys. Rev. 1961, 122, 827-830. [CrossRef]

21. Rhodes, B.L.; Legvold, S.; Spedding, F.H. Magnetic Properties of Holmium and Thulium Metals. Phys. Rev. 1958, 109, 1547-1550. [CrossRef]

22. Jennings, L.D.; Stanton, R.M.; Spedding, F.H. Heat Capacity of Terbium from 15 to $350^{\circ}$ K. J. Chem. Phys. 1957, $27,909-913$. [CrossRef]

23. Taylor, K.N.R.; Taylor, K.N.R.; Darby, M.I. Physics of Rare Earth Solids; Chapman and Hall: London, UK, 1972.

24. Methfessel, S.; Mattis, D.C. Magnetic Semiconductors; Springer: Berlin/Heidelberg, Germany, 1968.

25. Вонсовский, С.В.; Туров, Е.А. Об ОбменномВзаимодействииВалентных иВнутренних Электронов в Кристаллах(s-dОбменная МодельПереходных Кристаллов). ЖЭТф 1953, 24, 419-428. (In Russian)

26. Elliott, R.J.; Wedgwood, F.A. The Temperature Dependence of Magnetic Ordering in the Heavy Rare Earth Metals. Proc. Phys. Soc. 1964, 84, 63-75. [CrossRef]

27. Dzyaloshinskii, I.E. Character of phase transitions to a helical or sinusoidal state in magnetic materials. Sov. Phys. JETP 1977, 45, 1014-1022.

28. Dzyaloshinskii, I.E. The Theory of Helicoidal Structures in Antiferromagnets. II. Metals. Sov. Phys. JETP 1965, 20, $223-231$.

29. Evenson, W.E.; Liu, S.H. Theory of Magnetic Ordering in the Heavy Rare Earths. Phys. Rev. 1969, 178, 783-794. [CrossRef]

30. Evenson, W.E.; Liu, S.H. Generalized Susceptibilities and Magnetic Ordering of Heavy Rare Earths. Phys. Rev. Lett. 1968, 21, 432-434. [CrossRef]

31. Keeton, S.C.; Loucks, T.L. Electronic Structure of Rare-Earth Metals. I. Relativistic Augmented-Plane-Wave Calculations. Phys. Rev. 1968, 168, 672-678. [CrossRef]

32. Lu, J.-B.; Cantu, D.C.; Nguyen, M.-T.; Li, J.; Glezakou, V.-A.; Rousseau, R. Norm-Conserving Pseudopotentials and Basis Sets To Explore Lanthanide Chemistry in Complex Environments. J. Chem. Theory Comput. 2019, 15, 5987-5997; Erratum in 2020, $16,4754$. [CrossRef]

33. Jensen, J.B.; Mackintosh, A.R. Rare Earth Magnetism. Structures and Excitations; Clarendon Press: Oxford, UK, 1991.

34. Enkovaara, J.; Rostgaard, C.; Mortensen, J.J.; Chen, J.; Dułak, M.; Ferrighi, L.; Gavnholt, J.; Glinsvad, C.; Haikola, V.; Hansen, H.A.; et al. Electronic Structure Calculations with GPAW: A Real-Space Implementation of the Projector Augmented-Wave Method. J. Phys. Condens. Matter 2010, 22, 253202. [CrossRef]

35. Kresse, G.; Joubert, D. From Ultrasoft Pseudopotentials to the Projector Augmented-Wave Method. Phys. Rev. B 1999, 59, 1758-1775. [CrossRef]

36. Madsen, G.K.H.; Blaha, P.; Schwarz, K.; Sjöstedt, E.; Nordström, L. Efficient Linearization of the Augmented Plane-Wave Method. Phys. Rev. B 2001, 64, 195134. [CrossRef]

37. Kohn, W.; Sham, L.J. Self-Consistent Equations Including Exchange and Correlation Effects. Phys. Rev. 1965, 140, A1133-A1138. [CrossRef]

38. Perdew, J.P.; Zunger, A. Self-Interaction Correction to Density-Functional Approximations for Many-Electron Systems. Phys. Rev. B 1981, 23, 5048-5079. [CrossRef]

39. Monkhorst, H.J.; Pack, J.D. Special Points for Brillouin-Zone Integrations. Phys. Rev. B 1976, 13, 5188-5192. [CrossRef]

40. Freeman, A.J. Energy Band Structure, Indirect Exchange Interactions and Magnetic Ordering. In Magnetic Properties of Rare Earth Metals; Elliott, R.J., Ed.; Springer: Boston, MA, USA, 1972; pp. 245-333. ISBN 978-1-4757-5691-3.

41. Kurz, P.; Bihlmayer, G.; Blügel, S. Magnetism and Electronic Structure of Hcp Gd and the Gd(0001) Surface. J. Phys. Condens. Matter 2002, 14, 6353-6371. [CrossRef]

42. Tereshina, E.A.; Khmelevskyi, S.; Politova, G.; Kaminskaya, T.; Drulis, H.; Tereshina, I.S. Magnetic Ordering Temperature of Nanocrystalline Gd: Enhancement of Magnetic Interactions via Hydrogenation-Induced “Negative” Pressure. Sci. Rep. 2016, 6, srep22553. [CrossRef]

43. Sticht, J.; Kübler, J. Electronic Structure of Ferromagnetic Gd. Solid State Commun. 1985, 53, 529-532. [CrossRef]

44. Gimaev, R.R.; Zverev, V.I.; Mello, V.D. Magnetic Properties of Single-Crystalline Terbium and Holmium-Experiment and Modeling. J. Magn. Magn. Mater. 2020, 505, 166781. [CrossRef]

45. Elliott, R.J. Phenomenological Discussion of Magnetic Ordering in the Heavy Rare-Earth Metals. Phys. Rev. 1961, 124, 346-353. [CrossRef] 
46. Zverev, V.I.; Gimaev, R.R.; Komlev, A.S.; Kovalev, B.B.; Queiroz, F.G.; Mello, V.D. Magnetic Properties of Dysprosium-Experiment and Modeling. J. Magn. Magn. Mater. 2021, 524, 167593. [CrossRef]

47. Smart, J.S. Effective Field Theories of Magnetism; Saunders: Philadelphia, PA, USA, 1966.

48. Jensen, J. Hartree-Fock Renormalization of Magnetic Anisotropy in the Basal-Plane Ferromagnets Terbium and Dysprosium. J. Phys. C Solid State Phys. 1975, 8, 2769. [CrossRef]

49. Levy, P.M. Antisymmetric Exchange. Phys. Rev. Lett. 1968, 20, 1366-1370. [CrossRef]

50. Levy, P.M. Indirect Exchange via Spin-Orbit Coupled States. Solid State Commun. 1969, 7, 1813-1818. [CrossRef]

51. Elliott, R. (Ed.) Magnetic Properties of Rare Earth Metals; Springer: Boston, MA, USA, 1972; ISBN 978-1-4757-5693-7.

52. Yosida, K. Chapter V Magnetic Structures of Heavy Rare-Earth Metals. In Progress in Low Temperature Physics; Gorter, C.J., Ed.; Elsevier: Amsterdam, The Netherlands, 1964; Volume 4, pp. 265-295.

53. Sinha, S.K. Chapter 7 Magnetic structures and inelastic neutron scattering: Metals, alloys and compounds. In Handbook on the Physics and Chemistry of Rare Earths; Metals; Elsevier: Amsterdam, The Netherlands, 1978; Volume 1, pp. 489-589.

54. Sherrington, D. The Tilted Helix: An Intermediate Magnetic Phase. J. Phys. C Solid State Phys. 1973, 6, 1037-1053. [CrossRef]

55. Izyumov, Y.A. Modulated, or Long-Periodic, Magnetic Structures of Crystals. Sov. Phys. Uspekhi 1984, 27, 845-867. [CrossRef]

56. Jensen, J. Molecular Field Calculation of the Magnetic Structure in Erbium. J. Phys. F Met. Phys. 1976, 6, 1145. [CrossRef]

57. Habenschuss, M.; Stassis, C.; Sinha, S.K.; Deckman, H.W.; Spedding, F.H. Neutron Diffraction Study of the Magnetic Structure of Erbium. Phys. Rev. B 1974, 10, 1020-1026. [CrossRef]

58. Vonsovskii, S.V. Magnetism; Wiley: New York, NY, USA, 1974.

59. Cooper, B.R. Magnetic Properties of Rare Earth Metals. In Solid State Physics; Seitz, F., Turnbull, D., Ehrenreich, H., Eds.; Academic Press: Cambridge, MA, USA, 1968; Volume 21, pp. 393-490.

60. Garel, T.; Pfeuty, P. Commensurability Effects on the Critical Behaviour of Systems with Helical Ordering. J. Phys. C Solid State Phys. 1976, 9, L245-L249. [CrossRef]

61. Plumer, M.L.; Caillé, A. Magnetic Phase Diagrams of the Antiferromagnetic Planar Model on a Stacked Triangular Lattice. Phys. Rev. B 1990, 42, 10388-10396. [CrossRef]

62. Zverev, V.I.; Tishin, A.M.; Min, Z.; Mudryk, Y.; Gschneidner, K.A.; Pecharsky, V.K. Magnetic and Magnetothermal Properties, and the Magnetic Phase Diagram of Single-Crystal Holmium along the Easy Magnetization Direction. J. Phys. Condens. Matter 2015, 27, 146002. [CrossRef] [PubMed]

63. Lin, H.; Collins, M.F.; Holden, T.M.; Wei, W. Magnetic Structure of Erbium. Phys. Rev. B 1992, 45, 12873-12882. [CrossRef] [PubMed]

64. Watson, B.; Ali, N. On the Phase Diagram of Erbium. J. Alloy. Compd. 1997, 250, 662-665. [CrossRef]

65. Cooper, B.R. Magnetoelastic Effects and the Magnetic Properties of Rare-Earth Metals. Phys. Rev. Lett. 1967, 19, 900-903. [CrossRef]

66. Cooper, B.R. Spin Waves and Magnetic Resonance in Rare-Earth Metals: Thermal, Applied-Field, and Magnetoelastic Effects. Phys. Rev. 1968, 169, 281-294. [CrossRef]

67. Spichkin, Y.I.; Tishin, A.M. Magnetocaloric Effect at the First-Order Magnetic Phase Transitions. J. Alloy. Compd. 2005, 403, 38-44. [CrossRef]

68. Zverev, V.I.; Gimaev, R.R. Explanation of Relatively High Values of the Magnetic Entropy Change in Single Crystalline Terbium. Phys. B Condens. Matter 2016, 502, 187-189. [CrossRef]

69. Legvold, S. Chapter 3 Rare earth metals and alloys. In Handbook of Ferromagnetic Materials; Elsevier: Amsterdam, The Netherlands, 1980; Volume 1, pp. 183-295.

70. Fort, D.; Pecharsky, V.K.; Gschneidner, K.A. Solid State Electrotransport Purification of Dysprosium. J. Alloy. Compd. 1995, 226, 190-196. [CrossRef]

71. Zverev, V.I.; Tishin, A.M.; Chernyshov, A.S.; Mudryk, Y.; Gschneidner, K.A., Jr.; Pecharsky, V.K. Magnetic and Magnetothermal Properties and the Magnetic Phase Diagram of High Purity Single Crystalline Terbium along the Easy Magnetization Direction. J. Phys. Condens. Matter 2014, 26, 066001. [CrossRef]

72. Dan'kov, S.Y.; Tishin, A.M.; Pecharsky, V.K.; Gschneidner, K.A. Magnetic Phase Transitions and the Magnetothermal Properties of Gadolinium. Phys. Rev. B 1998, 57, 3478-3490. [CrossRef]

73. Chernyshov, A.S.; Tsokol, A.O.; Tishin, A.M.; Gschneidner, K.A.; Pecharsky, V.K. Magnetic and Magnetocaloric Properties and the Magnetic Phase Diagram of Single-Crystal Dysprosium. Phys. Rev. B 2005, 71, 184410. [CrossRef]

74. Pechan, M.J.; Stassis, C. Magnetic Structure of Holmium. J. Appl. Phys. 1984, 55, 1900-1902. [CrossRef]

75. Pecharsky, V.K.; Gschneidner, K.A.; Fort, D. Zero-Field and Magnetic-Field Low-Temperature Heat Capacity of Solid-State Electrotransport-Purified Erbium. Phys. Rev. B 1993, 47, 5063-5071. [CrossRef] [PubMed]

76. Nikitin, S.A.; Tishin, A.M.; Leontiev, P.I. Magnetocaloric Effect and Pressure Influence on Dysprosium Single Crystal Magnetization in the Range of Magnetic Phase Transition. J. Magn. Magn. Mater. 1991, 92, 405-416. [CrossRef]

77. Nikitin, S.A.; Tishin, A.M.; Red'ko, S.V. Magnetocaloric Effect in Single Crystals of Terbium and Its Alloys with Gadolinium. Phys. Met. Metallogr. 1990, 66, 77-85.

78. Andreenko, A.S.; Belov, K.P.; Nikitin, S.A.; Tishin, A.M. Magnetocaloric Effects in Rare-Earth Magnetic Materials. Sov. Phys. Uspekhi 1989, 32, 649. [CrossRef] 
79. Belov, K.P.; Levitin, R.Z.; Nikitin, S.A.; Ped'ko, A.V. Magnetic and Magnetoelastic Properties of Dysprosium and Gadolinium. Sov. Phys. JETP 1961, 13, 1096-1101.

80. Belov, K.P.; Levitin, R.Z.; Ponomarev, B.K. Magnetic and Magnetosriction Properties of an Erbiu Single Crystal in the Paramagnetic Region. JETP 1967, 24, 1101-1104.

81. Bartholin, H.; Bloch, D. Magnetic Ordering Temperatures of the Single Crystals of Rare Earth Metals at High Pressures: Gadolinium-Terbium-Dysprosium. J. Phys. Chem. Solids 1968, 29, 1063-1075. [CrossRef]

82. Alberts, L.; du Plessis, P.d.V. Thermal Expansion and Forced Magnetostriction in a Terbium Single Crystal. J. Appl. Phys. 1968, 39, 581-582. [CrossRef]

83. Darnell, F.J. Lattice Parameters of Terbium and Erbium at Low Temperatures. Phys. Rev. 1963, 132, 1098-1100. [CrossRef]

84. Ali, N.; Willis, F.; Steinitz, M.O.; Kahrizi, M.; Tindall, D.A. Observation of Transitions to Spin-Slip Structures in Single-Crystal Holmium. Phys. Rev. B 1989, 40, 11414-11416. [CrossRef] [PubMed]

85. Bates, S.; Patterson, C.; McIntyre, G.J.; Palmer, S.B.; Mayer, A.; Cowley, R.A.; Melville, R. The Magnetic Structure of Holmium. II. J. Phys. C Solid State Phys. 1988, 21, 4125-4141. [CrossRef]

86. Brits, G.H.F.; du Plessis, P.d.V. Criticalbehaviour of the Planar Helical to or from Paramagnetic Phase Transition in Dysprosium and Holmium. J. Phys. F Met. Phys. 1988, 18, 2659-2668. [CrossRef]

87. Cowley, R.A.; Bates, S. The Magnetic Structure of Holmium. I. J. Phys. C Solid State Phys. 1988, 21, 4113-4124. [CrossRef]

88. Alkhafaji, M.T.; Ali, N. Magnetic Phase Diagram of Dysprosium. J. Alloy. Compd. 1997, 250, 659-661. [CrossRef]

89. Andrianov, A.V.; Gaidukov, Y.P.; Vasil'ev, A.N.; Fawcett, E. The Magnetic Phase Diagrams of Dysprosium. J. Magn. Magn. Mater. 1991, 97, 246-250. [CrossRef]

90. Astrom, H.U.; Benediktsson, G. Magnetic Transitions in Dysprosium: A Calorimetric Study. J. Phys. F Met. Phys. 1988, 18, 2113-2120. [CrossRef]

91. Ali, N.; Willis, F. Magnetization of Single-Crystal Erbium. Phys. Rev. B 1990, 42, 6820-6822. [CrossRef]

92. Buck, S.; Fähnle, M. Ab Initio Calculation of the Giant Magnetostriction in Terbium and Erbium. Phys. Rev. B 1998, 57, R14044-R14047. [CrossRef]

93. Flippen, R.B. Changes in Magnetization of Single-Crystal Dysprosium, Erbium, and Holmium in High Magnetic Fields. J. Appl. Phys. 1964, 35, 1047-1048. [CrossRef]

94. Bagguley, D.M.S.; Liesegang, J.; Bleaney, B. Microwave Absorption Phenomena in Rare Earth Metals. Proc. R. Soc. Lond. Ser. A Math. Phys. Sci. 1967, 300, 497-518. [CrossRef]

95. Salamon, M.B.; Simons, D.S. Evidence for a Second Magnetic Phase Transition in Godolinium. Phys. Rev. B 1973, 7, $229-232$. [CrossRef]

96. Zverev, V.I.; Gimaev, R.R.; Tishin, A.M.; Mudryk, Y.; Gschneidner, K.A.; Pecharsky, V.K. The Role of Demagnetization Factor in Determining the 'True' Value of the Curie Temperature. J. Magn. Magn. Mater. 2011, 323, 2453-2457. [CrossRef]

97. Belov, K.P.; Ped'ko, A.V. “Helical” Antiferromagnetism of Gadolinium. Sov. Phys. JETP 1962, 15, 62-64.

98. Graham, C.D., Jr. Magnetocrystalline Anisotropy of Gadolinium. J. Phys. Soc. Jpn. 1962, 17, 1310. [CrossRef]

99. Cable, J.W.; Wollan, E.O. Neutron Diffraction Study of the Magnetic Behavior of Gadolinium. Phys. Rev. 1968, 165, 733-734. [CrossRef]

100. Will, G.; Nathans, R.; Alperin, H.A. Neutron Diffraction Investigation of a Gadolinium Single Crystal. J. Appl. Phys. 1964, 35, 1045-1046. [CrossRef]

101. Kataev, G.I.; Sattarov, M.R.; Tishin, A.M. Influence of Commensurability Effects on the Magnetic Phase Diagram of Terbium Single Crystals. Phys. Status Solidi A 1989, 114, K79-K82. [CrossRef]

102. Зверев, В.И. Магнитные и МагнитотепловыеСвойства Гадолиния, Тербия иГольмия в Области МагнитныхфазовыхПереходов. Ph.D. Thesis, МГУ им. М.В. Ломоносова, Moscow, Russia, 2012. (In Russian)

103. Herz, R.; Kronmüller, H. Field-Induced Magnetic Phase Transitions in Dysprosium. J. Magn. Magn. Mater. 1978, 9, $273-275$. [CrossRef]

104. Willis, F.; Ali, N. Magnetism in Single-crystal Dy below 10 K. J. Appl. Phys. 1991, 70, 6548-6549. [CrossRef]

105. Yu, J.; LeClair, P.R.; Mankey, G.J.; Robertson, J.L.; Crow, M.L.; Tian, W. Exploring the Magnetic Phase Diagram of Dysprosium with Neutron Diffraction. Phys. Rev. B 2015, 91, 014404. [CrossRef]

106. Astrom, H.U.; Nogues, J.; Nicolaides, G.K.; Rao, K.V.; Benediktsson, G. Magnetic Transitions in Single-Crystal Thulium. J. Phys. Condens. Matter 1991, 3, 7395-7402. [CrossRef]

107. Wakabayashi, N.; Cable, J.W.; Robertson, J.L. Magnetic Structures of Dy in Applied Fields. Phys. B Condens. Matter 1997, 241-243, 517-523. [CrossRef]

108. Jensen, J.; Mackintosh, A.R. Novel Magnetic Phases in Holmium. J. Magn. Magn. Mater. 1992, 104-107, 1481-1484. [CrossRef]

109. Kosevich, A.M.; Voronov, V.P.; Manzhos, I.V. Nonlinear Collective Excitations in an Easy Plane Magnet. Sov. Phys. JETP 1983, 57, 86-92.

110. Gor'kov, L.P.; Sokol, A.V. Kinetic Effects in Antiferromagnetic Conductors with Spin Density Waves. Sov. Phys. JETP 1987, 66, 1267-1273.

111. Koehler, W.C.; Cable, J.W.; Wilkinson, M.K.; Wollan, E.O. Magnetic Structures of Holmium. I. The Virgin State. Phys. Rev. 1966, 151, 414-424. [CrossRef] 
112. Koehler, W.C.; Cable, J.W.; Child, H.R.; Wilkinson, M.K.; Wollan, E.O. Magnetic Structures of Holmium. II. The Magnetization Process. Phys. Rev. 1967, 158, 450-461. [CrossRef]

113. Akhavan, M.; Blackstead, H.A. Magnetoresistance and Field-Induced Phase Transitions in the Helical and Conical States of Holmium. Phys. Rev. B 1976, 13, 1209-1215. [CrossRef]

114. Tindall, D.A.; Steinitz, M.O.; Plumer, M.L. Thermal Expansion in the Magnetically Ordered Phases of Holmium. J. Phys. F Met. Phys. 1977, 7, L263-L266. [CrossRef]

115. Jayasuriya, K.D.; Campbell, S.J.; Stewart, A.M. Specific Heat Study of a Holmium Single Crystal. J. Phys. F Met. Phys. 1985, 15, 225-239. [CrossRef]

116. Gibbs, D.; Moncton, D.E.; D’Amico, K.L.; Bohr, J.; Grier, B.H. Magnetic X-Ray Scattering Studies of Holmium Using Synchro-Tron Radiation. Phys. Rev. Lett. 1985, 55, 234-237. [CrossRef] [PubMed]

117. Bohr, J.; Gibbs, D.; Moncton, D.E.; D'Amico, K.L. Spin Slips and Lattice Modulations in Holmium: A Magnetic x-Ray Scattering Study. Phys. A 1986, 140, 349-358. [CrossRef]

118. Ellerby, M.; McEwen, K.A.; Jensen, J. Magnetoresistance and Magnetization Study of Thulium. Phys. Rev. B 1998, 57, 8416-8423. [CrossRef]

119. Fynbo, P.B. Magnetic Properties of Thulium. J. Phys. F Met. Phys. 1977, 7, 2179-2191. [CrossRef]

120. Zochowski, S.W.; McEwen, K.A. Magnetic Phase Diagram of Thulium. J. Magn. Magn. Mater. 1992, 104-107, 1515-1516. [CrossRef]

121. Gama, S.; Fóglio, M.E. Magnetization of Erbium in the Ordered and Paramagnetic Phases. Phys. Rev. B 1988, 37, $2123-2132$. [CrossRef]

122. Burger, J.P.; Vajda, P.; Daou, J.N.; Chouteau, G. Effect of Hydrogen in Solid Solution upon the Magnetic Properties of Monocrystalline Erbium. J. Phys. F Met. Phys. 1986, 16, 1275-1286. [CrossRef]

123. Atoji, M. Magnetic Structures of Er Single Crystal in 0-20 KOe Field. Solid State Commun. 1974, 14, 1047-1050. [CrossRef]

124. Elliott, J.F.; Legvold, S.; Spedding, F.H. Magnetic Properties of Erbium Metal. Phys. Rev. 1955, 100, 1595-1596. [CrossRef]

125. Willis, F.; Ali, N. Magnetization and Thermal Expansion of Single-crystal Er and Tm. J. Appl. Phys. 1991, 69, 5697-5699. [CrossRef]

126. Gibbs, D.; Bohr, J.; Axe, J.D.; Moncton, D.E.; D’Amico, K.L. Magnetic Structure of Erbium. Phys. Rev. B 1986, $34,8182-8185$. [CrossRef] [PubMed]

127. Bohr, J. Magnetic X-Ray Scattering: A New Tool for Magnetic Structure Investigations. J. Magn. Magn. Mater. 1990, 83, 530-534. [CrossRef]

128. Bohr, J.; Gibbs, D.; Axe, J.D.; Moncton, D.E.; D’amico, K.L.; Majkrzak, C.F.; Kwo, J.; Hong, M.; Chien, C.L.; Jensen, J. Diffraction Studies of Rare Earth Metals and Superlattices. Phys. B Condens. Matter 1989, 159, 93-105. [CrossRef]

129. Godovikov, S.K.; Kozin, M.G.; Turovtsev, V.V.; Shpinel, V.S. Hyperfine Fields Acting on Diamagnetic Impurities and the Anisotropic Exchange via the S- and d-Electrons in the Rare-Earth Metals. Phys. Status Solidi B 1976, 78, 103-111. [CrossRef]

130. Bogdanov, P.V.; Godovikov, S.K.; Kozin, M.G.; Moreva, N.I.; Shpinel, V.S. Features of the Hyperfine Interaction of Tin Impurities in Metallic Holmium. Hyperfine Interact. 1977, 5, 333-345. [CrossRef]

131. Irkhin, V.Y.; Irkhin, Y.P. Charge Screening and Magnetic Anisotropy in Metallic Rare-Earth Systems. Phys. Rev. B 1998, 57, 2697-2700. [CrossRef]

132. Brun, T.O.; Sinha, S.K.; Wakabayashi, N.; Lander, G.H.; Edwards, L.R.; Spedding, F.H. Temperature Dependence of the Periodicity of the Magnetic Structure of Thulium Metal. Phys. Rev. B 1970, 1, 1251-1253. [CrossRef]

133. Никитин, С.А. МагнитныеСвойства Редкоземельных Металлов и ИхСплавов; Publisher МГУ им. М.В. Ломоносова: Моsсоw, Russia, 1989. (In Russian)

134. Jordan, R.G.; Lee, E.W. Low-Temperature Magnetic Properties of Dysprosium Single Crystals. Proc. Phys. Soc. 1967, 92, 1074-1082. [CrossRef]

135. Schaub, B.; Mukamel, D. Phase Diagrams of Systems Exhibiting Incommensurate Structures. Phys. Rev. B 1985, 32, 6385-6393. [CrossRef] [PubMed]

136. Grazhdankina, N.P. Magnetic First Order Phase Transitions. Phys. Uspekhi 1969, 11, 727-745. [CrossRef]

137. Strukov, B.A. Global Hysteresis in Ferroelectrics with Incommensurate Phases. Phase Transit. 1989, 15, 143-179. [CrossRef]

138. Levanyuk, A.P.; Osipov, V.V.; Sigov, A.S.; Sobyanin, A.A. Change of Defect Structure and the Resultant Anomalies in the Properties of Substances near Phase-Transition Points. Sov. Phys. JETP 1979, 49, 176-188.

139. Woods, A.D.B.; Holden, T.M.; Powell, B.M. Observation of Spin Waves in Erbium. Phys. Rev. Lett. 1967, 19, 908-910. [CrossRef]

140. Stringfellow, M.W.; Holden, T.M.; Powell, B.M.; Woods, A.D.B. Spin-Wave Excitations in the Conical and Spiral Magnetic Phases of Holmium Metal. J. Appl. Phys. 1969, 40, 1443-1445. [CrossRef]

141. Nicklow, R.M.; Mook, H.A.; Smith, H.G.; Reed, R.E.; Wilkinson, M.K. Spin-Wave Dispersion Relation for Holmium in the Spiral Magnetic Phase. J. Appl. Phys. 1969, 40, 1452-1453. [CrossRef]

142. Møller, H.B.; Houmann, J.C.G.; Mackintosh, A.R. Magnetic Interactions in Rare-Earth Metals from Inelastic Neutron Scattering. Phys. Rev. Lett. 1967, 19, 312-314. [CrossRef]

143. Vorob'ev, V.V.; Krupotkin, M.Y.; Finkel', V.A. Magnetic phase transitions in dysprosium single crystals in weak magnetic fields. Sov. Phys. JETP 1988, 61, 1056-1059.

144. Bozorth, R.M. Magnetic Properties of Compounds and Solid Solutions of Rare-Earth Metals. J. Appl. Phys. 1967, 38 , $1366-1371$. [CrossRef] 
145. Weinstein, S.; Craig, R.S.; Wallace, W.E. Structural and Magnetic Characteristics of Dysprosium-Yttrium Solid Solutions. J. Appl. Phys. 1963, 34, 1354-1355. [CrossRef]

146. Child, H.R.; Cable, J.W. Magnetic Structure Properties of Gd-Y and Gd-Sc Alloys. J. Appl. Phys. 1969, 40, 1003-1005. [CrossRef]

147. Child, H.R.; Koehler, W.C.; Wollan, E.O.; Cable, J.W. Magnetic Properties of Heavy Rare Earths Diluted by Yttrium and Lutetium. Phys. Rev. 1965, 138, A1655-A1660. [CrossRef]

148. Bean, C.P.; Rodbell, D.S. Magnetic Disorder as a First-Order Phase Transformation. Phys. Rev. 1962, 126, 104-115. [CrossRef]

149. Rodbell, D.S.; Bean, C.P. Some Magnetic First-Order Transitions. J. Appl. Phys. 1962, 33, 1037-1041. [CrossRef]

150. Kittel, C. Model of Exchange-Inversion Magnetization. Phys. Rev. 1960, 120, 335-342. [CrossRef]

151. Landau, L.D.; Lifshitz, E.M. Statistical Physics, Part 1, 3rd ed.; Course of Theoretical Physics; Butterworth-Heinemann: Oxford, UK, 1980; Volume 5, ISBN 0-7506-3372-7.

152. Kuz'min, M.D. Landau-Type Parametrization of the Equation of State of a Ferromagnet. Phys. Rev. B 2008, 77, 184431. [CrossRef]

153. Zverev, V.I.; Tishin, A.M. Complex Behaviour of Magnetization and Magnetocaloric Effect in Low Magnetic Field in the Vicinity of Magnetic Phase Transitions. Available online: https:/ /ifiir.org/en/fridoc/complex-behaviour-of-magnetization-andmagnetocaloric-effect-in-low-26205 (accessed on 3 December 2020).

154. Skochdopole, R.E.; Griffel, M.; Spedding, F.H. Heat Capacity of Erbium from 15 to 320 K. J. Chem. Phys. 1955, $23,2258-2263$. [CrossRef]

155. Zimm, C.B.; Barclay, J.A.; Harkness, H.H.; Green, G.F.; Patton, W.G. Magnetocaloric Effect in Thulium. Cryogenics 1989, 29, 937-938. [CrossRef] 